\title{
Geometric conditions of a two-link-and-six muscle structure based on internal force stability
}

Hiroaki Ochi ${ }^{*}$, Hitoshi Kino ${ }^{2}$, Kenji Tahara $^{3}$ and Yuki Matsutani ${ }^{4}$

\begin{abstract}
In a musculoskeletal system, internal tensile forces are generated among the muscles because it is a redundant system. The balancing of the internal tensile forces for a given posture generates a potential field in the system. Therefore, the potential field is utilized for the sensorless feedforward position control and improvement of stability based on the feedback method. However, the stability of the internal tensile forces is strongly influenced by the muscular arrangement. Previous studies showed that a stable condition can be identified through the minimization of the potential at a desired posture, and the sufficient condition has been theoretically established; however, the geometric condition of the muscular arrangement has not been determined. To effectively exploit the characteristics of the internal tensile forces, the geometric condition must be elucidated in the design of a musculoskeletal system. This paper aims to clarify the geometric condition to generate stable internal tensile forces. Based on the conditions generating the potential that is minimum at the desired posture, the paper analyzes that the geometric condition on a musculoskeletal structure with two-link and six-muscle. Additionally, the identified condition is assessed based on simulations. As a result, we revealed the geometric condition of the muscular arrangement to generate stable internal tensile forces. By designing the muscular arrangement to satisfy the condition, the stability of the internal tensile forces is ensured, and consequently, a control method utilizing the characteristics of the internal tensile force is stably implemented.
\end{abstract}

Keywords: Musculoskeletal structure, Internal tensile force, Stability, Muscular arrangement, Design

\section{Introduction}

Human can generate a variety of adequate motions to adapt to their environs and specific tasks. The generation of robot motion that is akin to that of a human is one of the most important goals in robotics engineering.

To produce human-like motion, the mechanical structure of the human body should be investigated in detail. The human body possesses a musculoskeletal structure, which is composed of joints, muscles, and tendons that connect muscles to bones. Consequently, a human

\footnotetext{
*Correspondence: ochi@rs.socu.ac.jp

${ }^{1}$ Department of Mechanical Engineering, Faculty of Engineering, SanyoOnoda City University, 1-1-1, Daigaku-Dori, Sanyo-Onoda, Yamaguchi 756-0884, Japan

Full list of author information is available at the end of the article
}

generates voluntary motion by coordinated contraction of a muscle group. This is a redundant-driven-system because muscles and tendons can transmit only tensile forces to the skeletal structure. Because of this redundancy, the system is an ill-posed problem [1]. Moreover, the system can adjust the mechanical impedance of joints to adapt to its surrounding environment.

As an alternative approach, it considered the motor control in a human. Although the motor generation principle has not been clarified entirely; several generation hypotheses have been proposed. Bizz et al. proposed the "Equilibrium point (EP) hypothesis" where the equilibrium point between agonistic and antagonistic muscles is regulated [2]. Kawato et al. proposed a motor generation method that uses the inverse dynamics model acquired based on the feedback-error [3]. In these motor 
generation hypotheses, the primary focus is on the feedforward input from the brain as a crucial factor.

Based on the aforementioned, musculoskeletal structural systems and tendon-driven systems have been studied in the analysis of human motor control systems; for example, Kumamoto et al. [4] indicated that biarticular muscles that actuate two joints contribute to the achievement of human-like motion. Kozuki et al. [5] produced a musculoskeletal humanoid robot to address the analysis of human motion generation. In addition, some robotics systems were designed to be human-like and utilized characteristic advantages of the musculoskeletal structure; for example, by Koganezawa et al. [6], Mitsuda et al. [7], Ozawa et al. [8], Hitzmann et al. [9] and Shin et al. [10].

Kino et al. determined that the internal tensile forces among the muscles produce their own potential field that is strongly dependent on the muscular geometric arrangement, and that the characteristic can enable feedforward positioning by inputting balanced internal forces at the desired posture $[11,12]$. Expanding on this method, Matsutani et al. noted that the stability of the time delay, which affects the feedback-system, is improved by stable equilibrated internal tensile forces among the muscles [13]. These positioning methods that use internal tensile forces do not require complex calculations based on realtime and sensory feedback information like EP hypothesis. It is interesting that the positioning methods do not use muscular viscoelasticity unlike EP hypothesis; however, they utilize only characteristics of the muscular geometric arrangement.
Moreover, the feedforward positioning controller might be unstable depending on the position of the attachment point of a muscle. Kino et al. mathematically established a sufficient condition to achieve feedforward positioning control for a two-link musculoskeletal system driven manipulator with six muscles. This was achieved by analyzing the quasi-static potential using a secondorder Taylor expansion of the relationship between the joint angles and muscular lengths [14]. Based on the analysis, it was possible to determine the coefficient condition of the Taylor expansion.

Those studies revealed that the internal tensile forces have stability, and the stability depends on the position of the attachment point of a muscle. The internal tensile forces are used in the system; for example, adjustment of the mechanical impedance and stiffness, tensing mechanical muscles. When the structure generates the unstable internal tensile forces, the structural system negatively affects postural retainment and motion control even for feedback control systems. Therefore, the unstable muscular arrangement must be avoided in the system; however, the previous studies did not directly determine the muscular geometric arrangement condition, hence it is difficult to utilize this result in structural designs.

Expanding on the work [14], this study analyzes the stability of the internal tensile forces on the two-link and six-muscle musculoskeletal system as shown in Fig. 1, and clarifies the geometric condition for the muscular arrangement to achieve stable equilibrium at the desired posture. The result will be utilized in the design of a musculoskeletal system.

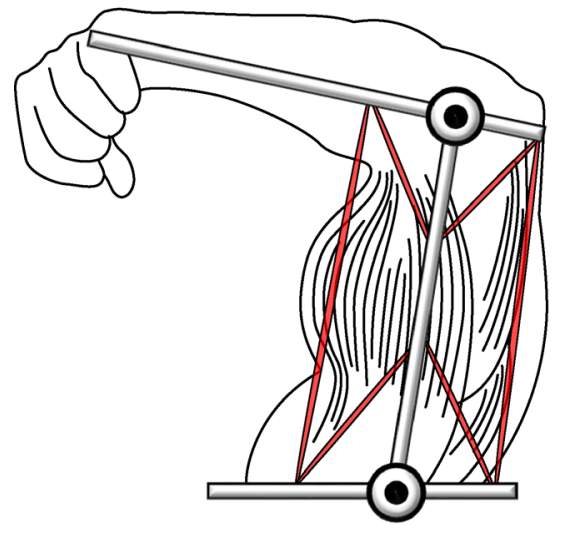

a Model imitating human upper arm

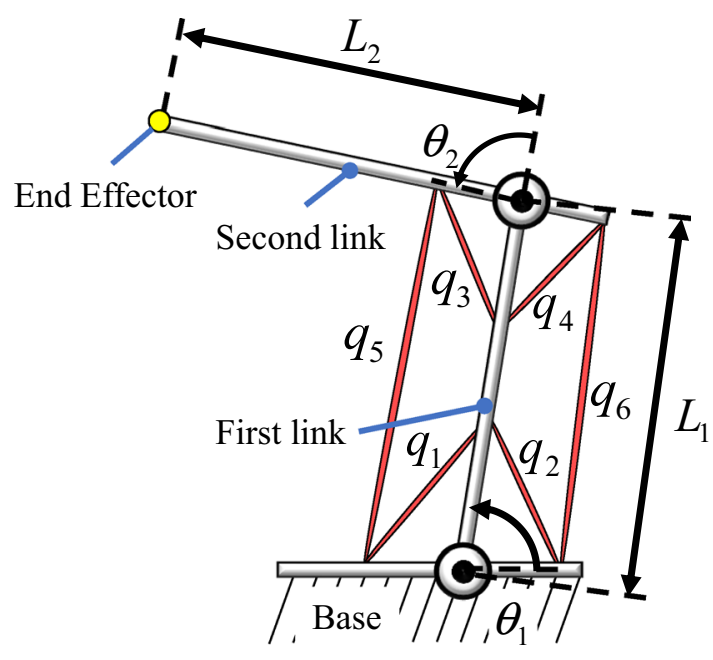

b Symbols of the model

Fig. 1 Musculoskeletal structural model (two-link with six-muscle) 
"Musculoskeletal system" section provides the details of the musculoskeletal system, kinematics, and internal tensile forces. "Stability of the internal tensile forces" section describes the stability analysis based on the quasi-static potential generated by internal tensile forces. "Analysis of geometric muscular arrangement conditions" section demonstrates the analysis of the geometric condition of the muscular arrangement based on the analysis of "Stability of the internal tensile forces" section. "Stability verification of geometric muscular arrangement conditions" section verifies the resultant condition through simulations. Finally, the conclusions are presented in "Conclusion" section.

\section{Musculoskeletal system Target model}

In the field of robotics, a musculoskeletal structure is utilized in human-like robots. The structure of these robotic systems consists of links instead of a skeletal system and utilize mechanical muscles such as actuator units to transfer tensile forces along wire cables $[5,8]$.

In this study, a mechanical muscle, such as a unit of an actuator and a wire cable, is called "muscle" as shown in Fig. 2. Additionally, the aspect of the musculoskeletal structure determined by fixing points of muscles on the links or base is called "muscular arrangement". The musculoskeletal system is a redundant-driven-system because it consists of antagonistic muscles, and it generates internal tensile forces among these muscles. In this study, the following assumptions are made for the target system:

- The system has two joints and six muscles as shown in Fig. 1. This model consists of four simple-joint

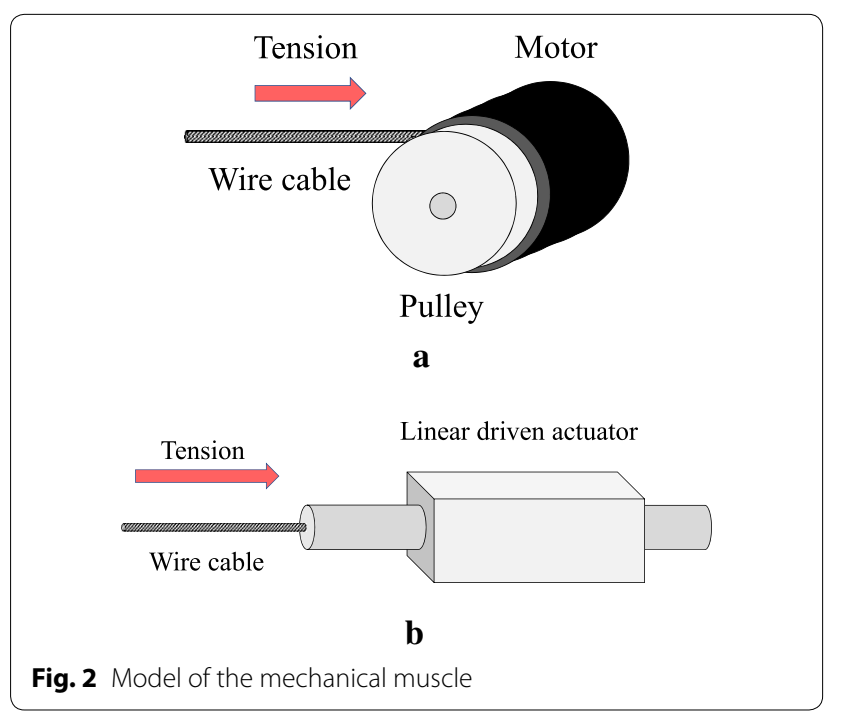

muscles (1-4) and two biarticular muscles (5 and 6). The joint angles are controlled by those six muscles and the resultant motion has a planar motion that is not affected by gravity.

- The target structure is designed based on a human upper arm. Therefore, the muscular arrangement does not differ significantly from that of a human musculoskeletal structure.

- Each muscle directly connects to points on the links/ base, and contract linearly. Furthermore, each muscle does not interfere with any mechanical parts.

- The muscles only transmit tensile forces but not pushing forces; they usually generate tensile forces and do not slack.

- In the presented analysis, the joint angles can move in the range from 0 [deg] to $180[\mathrm{deg}]$. Additionally, the posture satisfies "Vector Closure [15]" and it has an antagonistic muscular structure during motion. Non-singularity is satisfied during motion.

- The paper analyzes the quasi-static structural condition generating stable internal tensile forces; therefore, it does not especially consider dynamics parameters: the masses, the inertia, the viscoelasticity of the system.

\section{System static relations}

This section explains the static relations of the system. Table 1 shows the symbols of the musculoskeletal structural parameters using in the section. As shown in Fig. 1, the linkage lengths are $L_{1}, L_{2}$, and the muscular lengths are $q_{i}(i=1, \ldots, 6)$, respectively. $\boldsymbol{q}=\left(q_{1}, \ldots, q_{6}\right)^{T}$ represents the muscular length vector. The 1 st to 4 th muscles represent simple-joint muscles. The 5th and 6th muscles represent biarticular muscles. The tensile force generated on the $i$-th muscle is expressed as $\alpha_{i}$, and the tensile force vector is defined as $\boldsymbol{\alpha}=\left(\alpha_{1}, \ldots, \alpha_{6}\right)^{T}$. Each muscle generates only a tensile force, so $\alpha_{i}>0$ for all $i$ at any time. The tensile forces are positive in the pulling direction. The joint angles $\theta_{1}, \theta_{2}$ represent the shoulder and elbow

Table 1 Symbol list of the musculoskeletal structural parameter in the Section: System static relations

\begin{tabular}{ll}
\hline Symbol & Meaning \\
\hline$L_{1}, L_{2}$ & The linkage lengths \\
$i=1, \ldots, 6$ & The number of the muscles \\
$\boldsymbol{q}=\left(q_{1}, \ldots, q_{6}\right)^{T}$ & The muscular length vector \\
$\boldsymbol{\alpha}=\left(\alpha_{1}, \ldots, \alpha_{6}\right)^{T}$ & The tensile force vector \\
$\boldsymbol{\theta}=\left(\theta_{1}, \theta_{2}\right)^{T}$ & The joint angular vector \\
$\boldsymbol{\tau}=\left(\tau_{1}, \tau_{2}\right)^{T}$ & The joint torque vector \\
\hline
\end{tabular}


angles, where $\boldsymbol{\theta}=\left(\theta_{1}, \theta_{2}\right)^{T}$ is the joint angular vector. The joint torques $\tau_{1}, \tau_{2}$ represent the generated torques on the joints, where $\boldsymbol{\tau}=\left(\tau_{1}, \tau_{2}\right)^{T}$ is the joint torque vector.

The relation between the joint torque vector $\tau$ and the tensile force vector $\boldsymbol{\alpha}$ is expressed as follows:

$$
\tau=W(\theta) \boldsymbol{\alpha},
$$

where $\boldsymbol{W}(\boldsymbol{\theta}) \in \mathcal{R}^{2 \times 6}$ is a Jacobian matrix. The positive direction of the tensile force $\alpha_{i}$ is inverse to the positive direction of the muscular length $q_{i}$ in the equation.

$$
\boldsymbol{W}(\boldsymbol{\theta})=-\left(\frac{\partial \boldsymbol{q}}{\partial \boldsymbol{\theta}}\right)^{T} .
$$

The relationship between the muscular lengths and the joint angles are non-linear because the muscles are fixed on the link/base and driven linearly without any interfering mechanical parts, such as a guide-pully. Therefore, the Jacobian matrix $W$ is non-linearly changed by the joint angles $\boldsymbol{\theta}$.

The inverse relationship of Eq. (1) is written as follows:

$$
\boldsymbol{\alpha}=W^{+}(\boldsymbol{\theta}) \tau+\boldsymbol{v}(\boldsymbol{\theta})
$$

where $W^{+}(\boldsymbol{\theta})$ is the pseudo inverse matrix defined as $\boldsymbol{W}^{+}(\boldsymbol{\theta})=\boldsymbol{W}^{T}(\boldsymbol{\theta})\left(\boldsymbol{W}(\boldsymbol{\theta}) \boldsymbol{W}^{T}(\boldsymbol{\theta})\right)^{-1}$. The second term $\boldsymbol{v}(\boldsymbol{\theta})$ represents the internal tensile force among the muscles that belongs to the null-space of $\boldsymbol{W}(\boldsymbol{\theta})$ at the posture $\boldsymbol{\theta}$, and is expressed as

$$
\boldsymbol{v}(\boldsymbol{\theta})=\left(\boldsymbol{I}-\boldsymbol{W}^{+}(\boldsymbol{\theta}) \boldsymbol{W}(\boldsymbol{\theta})\right) \boldsymbol{k}_{\boldsymbol{e}},
$$

where $\boldsymbol{k}_{\boldsymbol{e}} \in \mathcal{R}^{6 \times 1}$ is determined arbitrarily.

\section{Stability of the internal tensile forces}

Relationship between internal tensile forces and potential

This section considers the case of a target system in which the input internal tensile force $\boldsymbol{v}_{\boldsymbol{d}}$ balances a desired posture $\boldsymbol{\theta}_{\boldsymbol{d}}$ as follows:

$$
\alpha=v_{d},
$$

where,

$$
\boldsymbol{v}_{d}=\boldsymbol{v}\left(\boldsymbol{\theta}_{d}\right)=\left(I-W^{+}\left(\boldsymbol{\theta}_{d}\right) W\left(\boldsymbol{\theta}_{d}\right)\right) \boldsymbol{k}_{e} .
$$

The arbitrary numbers in the vector $\boldsymbol{k}_{\boldsymbol{e}}$ are selected such that the muscles do not slack as follows:

$$
v_{d i}>0 \text { for any } i(i=1, \ldots, 6) \text {. }
$$

In a later analysis, the vector $\boldsymbol{k}_{\boldsymbol{e}}$ is defined as a constant vector.

When $\boldsymbol{\alpha}=\boldsymbol{v}_{\boldsymbol{d}}$, a joint torque is not generated at the desired posture $\boldsymbol{\theta}_{\boldsymbol{d}}$ as shown in the follows:

$$
\boldsymbol{\tau}=W\left(\boldsymbol{\theta}_{d}\right) \boldsymbol{v}_{\boldsymbol{d}}=\mathbf{0} .
$$

However, at another posture $\boldsymbol{\theta} \neq \boldsymbol{\theta}_{\boldsymbol{d}}$, the joint torque is generated as follows:

$$
\tau=W(\theta) v_{d} \neq \mathbf{0}
$$

When the torque is generated for the desired posture, the internal tensile forces are stable at the desired posture. However, when the torque is generated for a different posture from that of the desired one, the internal tensile forces are unstable at the desired posture.

Kino et al. indicated that the internal tensile force $\boldsymbol{v}_{\boldsymbol{d}}$ generates a particular potential field, according to the characteristics described in the preceding section; therefore, the internal tensile forces are stable when the quasistatic potential defined as follows is minimized at the desired posture $[11,12]$ :

$$
P(\boldsymbol{\theta})=\left(\boldsymbol{q}(\boldsymbol{\theta})-\boldsymbol{q}\left(\boldsymbol{\theta}_{\boldsymbol{d}}\right)\right)^{T} \boldsymbol{v}_{\boldsymbol{d}} .
$$

By using the stability of the internal tensile forces, they achieved feedforward positioning control by inputting constant tensile forces in the muscles as Eq. (5), and subsequently analyzed the dynamic stability of the feedforward positioning controlled system with two-link and six-muscle by using the Lyapunov function [14]. The control method is simply used to input constant equilibrium tensile forces at the desired posture. Therefore, the system can be controlled without any displacement feedback or complex real-time computing [13].

The previous analysis revealed that the shape of the potential $P(\boldsymbol{\theta})$ that influences the stability of the internal tensile forces is sensitive to the muscular arrangement [12]. For example, in the case of two-muscular systems as shown as Fig. 3a and b, the arrangement Fig. 3a has a little offset $d$ to the muscle fixed points. However, the arrangement Fig. 3b does not have the offset $d$.

Figure 4 represents the resultant shapes of the potential for the desired posture $\boldsymbol{\theta}_{\boldsymbol{d}}=(90,90)^{T}$ [deg] and $\boldsymbol{k}_{\boldsymbol{e}}=(1 \ldots 1)^{T}$ on the muscular arrangements Fig. 3a and b. As shown in Fig. 4a, the shape of the potential $P$ is minimized at the desired posture $\boldsymbol{\theta}_{\boldsymbol{d}}$ for the arrangement $3 \mathrm{a}$. Therefore, the internal tensile forces have stability, and they generate convergent torque at the desired posture $\boldsymbol{\theta}_{\boldsymbol{d}}$. However, as shown in Fig. $4 \mathrm{~b}$, the potential of the arrangement Fig. $3 \mathrm{~b}$ is not minimized at the desired posture; hence, the internal tensile forces are unstable, and they do not generate convergent torque at the desired posture $\boldsymbol{\theta}_{\boldsymbol{d}}$.

This example demonstrates that the shape of the potential $P$ is strongly dependent on the muscular arrangement. The system's stability and convergence at the desired posture are similarly affected by the shape of 


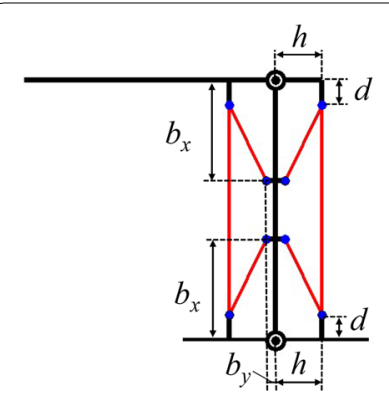

a Stable arrangement

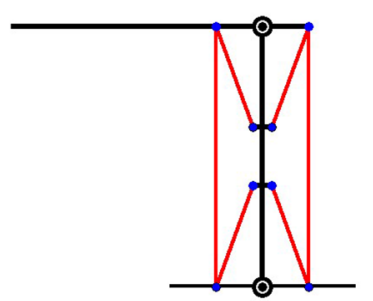

b Unstable arrangement $L_{1}=310, L_{2}=270, h=50, b_{x}=120, b_{y}=10, d=30$ or $\left.0[\mathrm{~mm}]\right)$

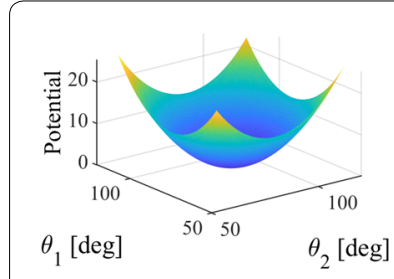

a Stable potential

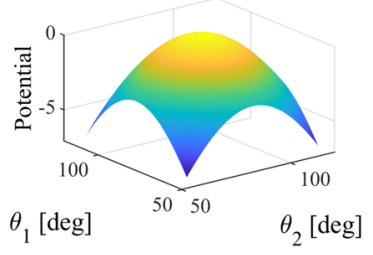

b Unstable potential
Fig. 4 Shapes of the potential (desired angle $\boldsymbol{\theta}_{\boldsymbol{d}}=(90,90)^{T}[\mathrm{deg}$, $\left.\boldsymbol{k}_{\boldsymbol{e}}=(1, \ldots, 1)^{T}\right)$

the potential. Although only the case of $\boldsymbol{\theta}_{\boldsymbol{d}}=(90,90)$ [deg] is shown as an example, the other cases at arbitrarily desired postures in the possible motion range exhibit the same characteristic for the muscular arrangements Fig. 3a and b [12].

\section{Stable condition for two-link and six-muscle system}

As described in the preceding section, the sufficient condition for which the muscular internal tensile forces are locally stable at the desired posture $\boldsymbol{\theta}_{\boldsymbol{d}}$ is that the potential $P(\boldsymbol{\theta})$ is minimized. By approximating the potential $P(\theta)$ by using a second-order Taylor expansion for the relationship between the joint angle $\boldsymbol{\theta}$ and the muscular length $\boldsymbol{q}$, Kino et al. determined the stable condition of the coefficients [14].

This study aims to identify the geometric condition of the muscular arrangement by expanding the Kino's stable condition. Succeeding part elucidates the Kino's stable condition because of important principle in this study.
The paper considers the stability of internal tensile forces in the vicinity of the desired posture $\boldsymbol{\theta}_{\boldsymbol{d}}$. When the potential $P(\boldsymbol{\theta})$ has a local minimum at the desired posture $\boldsymbol{\theta}_{\boldsymbol{d}}$, the following two conditions are obtained:

$$
\begin{aligned}
& \left.\frac{\partial P(\boldsymbol{\theta})}{\partial \boldsymbol{\theta}}\right|_{\boldsymbol{\theta}=\boldsymbol{\theta}_{d}}=-\left.\boldsymbol{W}(\boldsymbol{\theta}) \boldsymbol{v}_{d}\right|_{\boldsymbol{\theta}=\boldsymbol{\theta}_{d}}=\mathbf{0}, \\
& \left.\frac{\partial^{2} P(\boldsymbol{\theta})}{\partial \boldsymbol{\theta}^{2}}\right|_{\boldsymbol{\theta}=\boldsymbol{\theta}_{d}}=\boldsymbol{H}\left(\boldsymbol{\theta}_{d}\right)>\mathbf{0} .
\end{aligned}
$$

Equation (11) is always satisfied based on the definition of the internal tensile force vector $\boldsymbol{v}_{\boldsymbol{d}}$, which is a vector that belongs to the null-space of $\boldsymbol{W}\left(\boldsymbol{\theta}_{\boldsymbol{d}}\right)$. Therefore, the local minimum condition of the potential $P(\theta)$ at the desired posture $\boldsymbol{\theta}_{\boldsymbol{d}}$ only depends on the positive definiteness of the hessian matrix $\boldsymbol{H}\left(\boldsymbol{\theta}_{\boldsymbol{d}}\right) \in \mathcal{R}^{2 \times 2}$ described in Eq. (12). The necessary and sufficient condition that the Hessian matrix $\boldsymbol{H}\left(\boldsymbol{\theta}_{\boldsymbol{d}}\right)$ has a positive definiteness is equivalent to the follows:

$$
\begin{aligned}
& \operatorname{trace}\left(\boldsymbol{H}\left(\boldsymbol{\theta}_{\boldsymbol{d}}\right)\right)>0, \\
& \left|\boldsymbol{H}\left(\boldsymbol{\theta}_{\boldsymbol{d}}\right)\right|>0 .
\end{aligned}
$$

The conditional Eqs. (13) and (14) are analyzed based on the coefficients of the approximated muscular length $q_{i}^{*}$, calculated using the second-order Taylor expansion of the relationship between the joint angle $\boldsymbol{\theta}$ and the muscular length $q_{i}$ near $\boldsymbol{\theta}^{*}$. The approximated muscular length $q_{i}^{*}$ in the vicinity of $\boldsymbol{\theta}=\boldsymbol{\theta}^{*}$ is defined as follows:

$$
\begin{aligned}
q_{i}^{*}(\boldsymbol{\theta})= & q_{i}\left(\boldsymbol{\theta}^{*}\right)+\boldsymbol{Q}_{\mathbf{1}_{i}}\left(\boldsymbol{\theta}^{*}\right)\left(\boldsymbol{\theta}-\boldsymbol{\theta}^{*}\right) \\
& +\frac{1}{2}\left(\boldsymbol{\theta}-\boldsymbol{\theta}^{*}\right)^{T} \boldsymbol{Q}_{\mathbf{2}_{i}}\left(\boldsymbol{\theta}^{*}\right)\left(\boldsymbol{\theta}-\boldsymbol{\theta}^{*}\right) .
\end{aligned}
$$

In the analysis, the range of $\boldsymbol{\theta}^{*}=\left(\theta_{1}^{*}, \theta_{2}^{*}\right)^{T}$ is defined as $0<\theta_{1}^{*}<180$ [deg], $0<\theta_{2}^{*}<180$ [deg] and satisfies "Vector Closure" similarly to the range of the joint angle $\boldsymbol{\theta}$. $\boldsymbol{Q}_{1}\left(\boldsymbol{\theta}^{*}\right)$ and $\boldsymbol{Q}_{2_{i}}\left(\boldsymbol{\theta}^{*}\right)$ are the coefficient matrices defined as follows:

$$
\begin{aligned}
\boldsymbol{Q}_{\mathbf{1}_{i}}\left(\boldsymbol{\theta}^{*}\right) & =\left(\epsilon_{1 i}, \epsilon_{2 i}\right), \\
\boldsymbol{Q}_{\boldsymbol{2}_{i}}\left(\boldsymbol{\theta}^{*}\right) & =\left[\begin{array}{ll}
a_{i} & b_{i} \\
b_{i} & c_{i}
\end{array}\right] \\
& =\left.\left[\begin{array}{ll}
\frac{\partial^{2} q_{i}(\boldsymbol{\theta})}{\partial \theta_{1}^{2}} & \frac{\partial^{2} q_{i}(\boldsymbol{\theta})}{\partial \theta_{2} \partial \theta_{2}} \\
\frac{\partial^{2} q_{i}(\boldsymbol{\theta})}{\partial \theta_{1} \partial \theta_{2}} & \frac{\partial^{2} q_{i}(\boldsymbol{\theta})}{\partial \theta_{2}^{2}}
\end{array}\right]\right|_{\boldsymbol{\theta}=\boldsymbol{\theta}^{*}} .
\end{aligned}
$$

Substituting the approximated muscular length $q_{i}^{*}$ $(i=1, \ldots, 6)$, the conditional Eqs. (13) and (14) are approximately rewritten as follows: 
$\operatorname{trace}\left(H^{*}\right)=\boldsymbol{u} \boldsymbol{v}_{\boldsymbol{d}}$

$$
\begin{aligned}
\left|\boldsymbol{H}^{*}\right| & =\boldsymbol{v}_{\boldsymbol{d}}^{T} \boldsymbol{N} \boldsymbol{v}_{\boldsymbol{d}}, \\
\boldsymbol{u} & =\left(a_{1}, a_{2}, c_{3}, c_{4}, a_{5}+c_{5}, a_{6}+c_{6}\right), \\
\boldsymbol{N} & =\left(\begin{array}{llllll}
0 & 0 & n_{13} & n_{14} & n_{15} & n_{16} \\
0 & 0 & n_{23} & n_{24} & n_{25} & n_{26} \\
0 & 0 & 0 & 0 & n_{35} & n_{36} \\
0 & 0 & 0 & 0 & n_{45} & n_{46} \\
0 & 0 & 0 & 0 & n_{55} & n_{56} \\
0 & 0 & 0 & 0 & 0 & n_{66}
\end{array}\right)
\end{aligned}
$$

where, $n_{13}=a_{1} c_{3}, n_{14}=a_{1} c_{4}, n_{15}=a_{1} c_{5}, n_{16}=a_{1} c_{6}$, $n_{23}=a_{2} c_{3}, \quad n_{24}=a_{2} c_{4}, \quad n_{25}=a_{2} c_{5}, \quad n_{26}=a_{2} c_{6}$, $n_{35}=a_{5} c_{3}, \quad n_{36}=a_{6} c_{3}, \quad n_{45}=a_{5} c_{4}, \quad n_{46}=a_{6} c_{4}$, $n_{55}=a_{5} c_{5}-b_{5}^{2}, \quad n_{56}=a_{5} c_{6}+a_{6} c_{5}-2 b_{5} b_{6}$, $n_{66}=a a_{6} c_{6}-b_{6}^{2} . \quad v_{d i} \quad$ is always satisfied as $v_{d i}>0(i=1 \ldots 6)$ from Eq. (7). Therefore, when all the numbers in the vector $\boldsymbol{u}$ and the matrix $\boldsymbol{N}$ in Eqs. (18) and (19) are positive, the conditional Eqs. (13) and (14) are sufficiently satisfied. As a result, the potential $P(\boldsymbol{\theta})$ is locally invariably minimized at the desired posture $\boldsymbol{\theta}_{\boldsymbol{d}}$.

According to the preceding results, the internal tensile forces $\boldsymbol{v}_{\boldsymbol{d}}$ are stable at the desired posture $\boldsymbol{\theta}_{\boldsymbol{d}}$ because it sufficiently satisfies Eqs. (13) and (14) based on the second-order approximation, when each coefficient $a_{j}, c_{k}$, $b_{5}, b_{6}(j=1,2,5,6, k=3,4,5,6)$ defined as the approximated muscular length $q_{i}^{*}$ is satisfied as follows:

Stable conditions of the internal tensile forces when using Taylor expansion coefficient in the neighborhood of the desired posture $\theta_{d}$.

$$
\begin{aligned}
& a_{j}>0(j=1,2,5,6), \\
& c_{k}>0(k=3,4,5,6), \\
& a_{5} c_{5}>b_{5}^{2}, \\
& a_{6} c_{6}>b_{6}^{2} .
\end{aligned}
$$

The number $a_{5} c_{6}+a_{6} c_{5}-2 b_{5} b_{6}$ of the 5 th row and 6 th column in the matrix $N$ is positive when the conditions (20)-(23) are satisfied (see Appendix A). The most important finding in this analysis is that the conditions (20)-(23) never contain any information on a desired posture $\boldsymbol{\theta}_{\boldsymbol{d}}$; that is available for any desired posture near $\boldsymbol{\theta}^{*}$ when the conditions are satisfied.

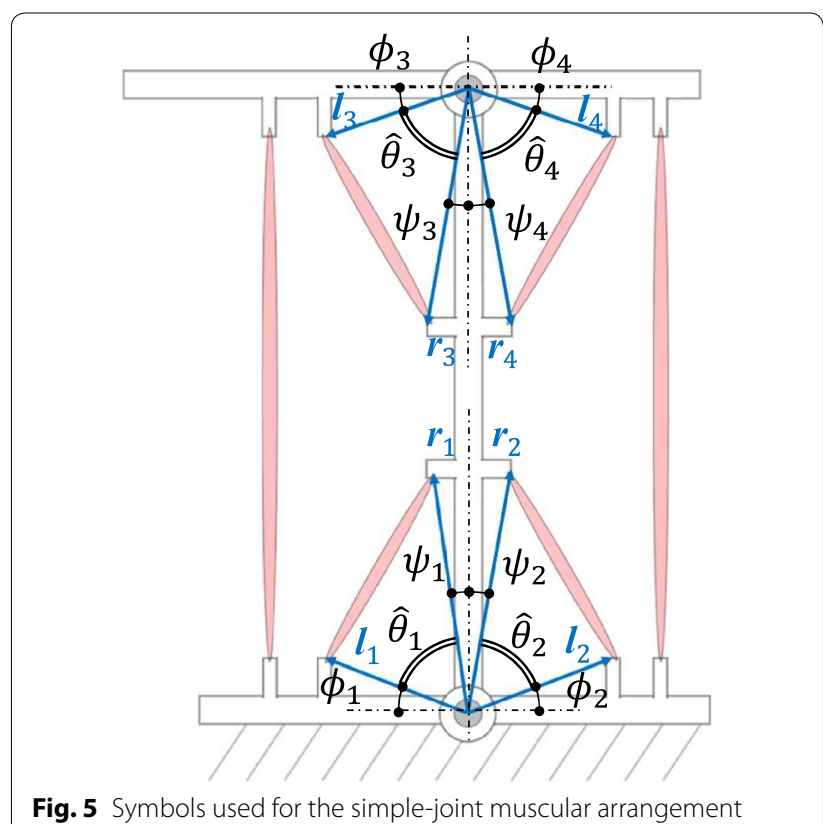

Table 2 Symbol list of the simple-joint muscular arrangement

\begin{tabular}{ll}
\hline Symbol & Meaning \\
\hline$\hat{i}=1,2,3,4$ & The number of the simple-joint muscle \\
$\boldsymbol{l}_{\hat{i}}, \boldsymbol{r}_{\hat{i}}$ & $\begin{array}{r}\text { The vectors from the rotational center of the driven joint } \\
\text { to the muscular fixed points }\end{array}$ \\
$\hat{k}_{\hat{i}}, r_{\hat{i}}$ & $\begin{array}{l}\text { The lengths of the vector } \boldsymbol{l}_{\hat{i}} \text { and } \boldsymbol{r}_{\hat{i}} \\
\phi_{\hat{i}}, \psi_{\hat{i}}\end{array}$ \\
$\hat{\theta}_{\hat{i}}$ & The angles between the base/link and the vectors $\boldsymbol{l}_{\hat{i}}$ or $\boldsymbol{r}_{\hat{i}}$ \\
\hline
\end{tabular}

\section{Analysis of geometric muscular arrangement conditions}

The stable conditions of internal tensile forces highlighted in the preceding section (20)-(23) described in the reference [14] are merely the conditions of the coefficients for the Taylor-extended muscular length using the joint angle $\boldsymbol{\theta}$. However, these conditions do not directly indicate how to arrange the muscles to generate stable internal tensile forces. This section expands the conditions (20)-(23), and establishes the geometric muscular arrangement conditions for the two-link, six-muscle system.

\section{Geometric condition for simple-joint muscles}

Initially, the simple-joint muscles represented as 1st-4th muscles in Fig. 1 are analyzed. The arranged parameters of the simple-joint muscles $\hat{i}(\hat{i}=1,2,3,4)$ are defined in Fig. 5. Table 2 shows the symbols of the simple-joint muscular arrangement. $\boldsymbol{l}_{\hat{i}}$ and $\boldsymbol{r}_{\hat{i}}$ are the vectors from 
the rotational center of the driven joint by the $i$-th muscle, to the muscular fixed points on the base/link and the vector lengths are $l_{\hat{i}}$ and $r_{\hat{i}}$ respectively. The angles $\phi_{\hat{i}}, \psi_{\hat{i}}$ are defined between the base/link and the vectors $\boldsymbol{l}_{\hat{\boldsymbol{i}}}$ or $\boldsymbol{r}_{\hat{\boldsymbol{i}}}$ respectively. The angles $\hat{\theta}_{\hat{i}}$ are defined between the vector $\boldsymbol{l}_{\hat{i}}$ and the vector $\boldsymbol{r}_{\hat{i}} . l_{\hat{i}}, r_{\hat{i}}, \phi_{\hat{i}}, \psi_{\hat{i}}$ are constant even if the joint angles $\theta_{1}$ and $\theta_{2}$ are changed. Moreover, $\hat{\theta}_{\hat{i}}$ is defined by $\phi_{\hat{i}}, \psi_{\hat{i}}$ and the joint angle $\theta$, thus, $\hat{\theta}_{\hat{i}}$ changes during angular motion. The muscle length $q_{\hat{i}}$ is expressed using the cosine formula as follows:

$$
q_{\hat{i}}=\sqrt{l_{\hat{i}}^{2}+r_{\hat{i}}^{2}-2 l_{\hat{i}} r_{\hat{i}} \cos \hat{\theta}_{\hat{i}}}(\hat{i}=1,2,3,4) .
$$

For the stable conditions (20)-(23), the conditions of the simple-joint muscle relate to only $a_{\hat{i}}>0$ and $c_{\hat{i}}>0$ as described in Eqs. (20) and (21). Here, the geometric muscular arrangement condition is analyzed by focusing on the 1st simple-joint muscle. $a_{1}$ is defined as $a_{1}=\left.\frac{\partial^{2} q_{1}(\boldsymbol{\theta})}{\partial \theta_{1}^{2}}\right|_{\boldsymbol{\theta}=\theta^{*}}$ from Eq. (17), and rewritten as the follows from Eq. (24):

$$
\begin{aligned}
a_{1} & =\left.\frac{\partial^{2} q_{1}}{\partial \theta_{1}^{2}}\right|_{\boldsymbol{\theta}=\boldsymbol{\theta}^{*}} \\
& =-\frac{r_{1} l_{1}\left(r_{1} \cos \hat{\theta}_{1}^{*}-l_{1}\right)\left(l_{1} \cos \hat{\theta}_{1}^{*}-r_{1}\right)}{q_{1}^{* 3}},
\end{aligned}
$$

where $\hat{\theta}_{1}^{*}$ and $q_{1}^{*}$ are the constant values of $\hat{\theta}_{1}$ and $q_{1}$ under $\boldsymbol{\theta}=\boldsymbol{\theta}^{*}$, which is the reference point for the Taylor expansion. From the definitions of that $q_{1}^{*}, l_{1}$ and $r_{1}$ are positive real numbers, $\left(r_{1} \cos \hat{\theta}_{1}^{*}-l_{1}\right)\left(l_{1} \cos \hat{\theta}_{1}^{*}-r_{1}\right)$ is negative when the Eq. (25) is satisfied $a_{1}>0$; therefore, $r_{1} \cos \hat{\theta}_{1}^{*}-l_{1}$ and $l_{1} \cos \hat{\theta}_{1}^{*}-r_{1}$ have the different sign. As a result, the condition (20) is rewritten either the following conditions:

$$
\begin{aligned}
\text { Condition }(\mathrm{A}) & =l_{1} \cos \hat{\theta}_{1}^{*}>r_{1} \text { and } r_{1} \cos \hat{\theta}_{1}^{*}<l_{1}, \\
\text { Condition }(\mathrm{B}) & =l_{1} \cos \hat{\theta}_{1}^{*}>r_{1} \text { and } r_{1} \cos \hat{\theta}_{1}^{*}<l_{1} .
\end{aligned}
$$

For Condition (A), it is necessary that $l_{1}>r_{1}$ to satisfy $l_{1} \cos \hat{\theta}_{1}^{*}>r_{1}$; therefore $r_{1} \cos \hat{\theta}_{1}^{*}<l_{1}$ is always satisfied by considering $r_{1}>0$ and $\max \left\{\cos \hat{\theta}_{1}^{*}\right\}=1$. Similarly, for the condition (B), it is necessary that $r_{1}>l_{1}$ to satisfy $r_{1} \cos \hat{\theta}_{1}^{*}>l_{1}$; therefore $l_{1} \cos \hat{\theta}_{1}^{*}<r_{1}$ is satisfied. As a result, Conditions (A) and (B) are rewritten as Conditions $\left(\mathrm{A}^{\prime}\right)$ and $\left(\mathrm{B}^{\prime}\right)$ as follows:

$$
\begin{array}{ll}
\operatorname{Condition}\left(\mathrm{A}^{\prime}\right): & l_{1} \cos \hat{\theta}_{1}^{*}>r_{1}, \\
\operatorname{Condition}\left(\mathrm{B}^{\prime}\right): & r_{1} \cos \hat{\theta}_{1}^{*}>l_{1},
\end{array}
$$

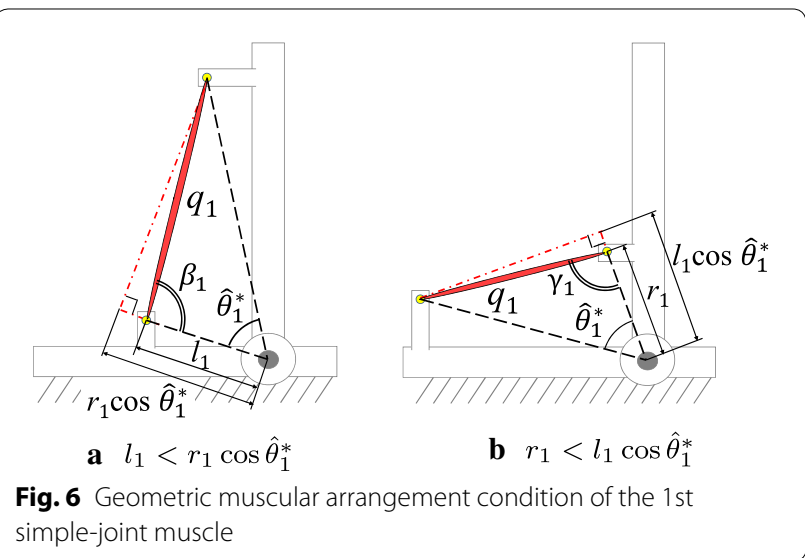

The design method for the muscular arrangement of the 1st simple-joint muscle is then considered from the preceding conditions ( $\left.\mathrm{A}^{\prime}\right)$ and $\left(\mathrm{B}^{\prime}\right)$. Figure 6a displays the muscular arrangement that satisfies Condition $\left(\mathrm{A}^{\prime}\right)$. To satisfy this condition the length $l_{1}$ must be shorter than the length $r_{1} \cos \hat{\theta}_{1}^{*}$ that projects $\boldsymbol{r}_{1}$ onto the vector $l_{1}$. Considering the triangle consisting of the vector $\boldsymbol{l}_{1}, \boldsymbol{r}_{1}$ and the 1 st muscle, the geometric condition is reworded such that the angle $\beta_{1}\left(0<\beta_{1}<180\right.$ [deg]) consisting of the vector $l_{1}$ and the 1 st muscle, must be an obtuse angle.

Similarly, Fig. 6b represents the muscular arrangement that satisfies Condition ( $\left.\mathrm{B}^{\prime}\right)$. The geometric condition indicates that the angle $\gamma_{1}$ consisting of the vector $\boldsymbol{r}_{1}$ and the 1st muscle must be an obtuse angle. As a result, the geometric muscular arrangement condition for the 1st muscle is expressed as follows:

$$
90<\beta_{1}<180 \text { or } 90<\gamma_{1}<180 \text { [deg]. }
$$

The conditions for the other simple-joint muscles $a_{2}>0$, $c_{3}>0, c_{4}>0$ are similarly analyzed and are obtained as with the 1st muscle.

Consequently, the simple-joint muscular arrangement required to generate stable internal tensile forces at a desired posture must be satisfied with either of the following conditions:

\section{The geometric conditions of the simple-joint muscular arrangement}

The geometric muscular arrangement conditions required to satisfy $a_{1}>0, a_{2}>0, c_{3}>0, c_{4}>0$ in Eqs. (20) and (21) are that the angles, $\beta_{\hat{i}}$ and $\gamma_{\hat{i}}$, defined by the arrangement of the simple-joint muscles $\hat{i}$-th $(\hat{i}=1,2,3,4)$ must be satisfied either of the following conditions:

$$
90<\beta_{\hat{i}}<180 \text { or } 90<\gamma_{\hat{i}}<180 \text { [deg]. }
$$




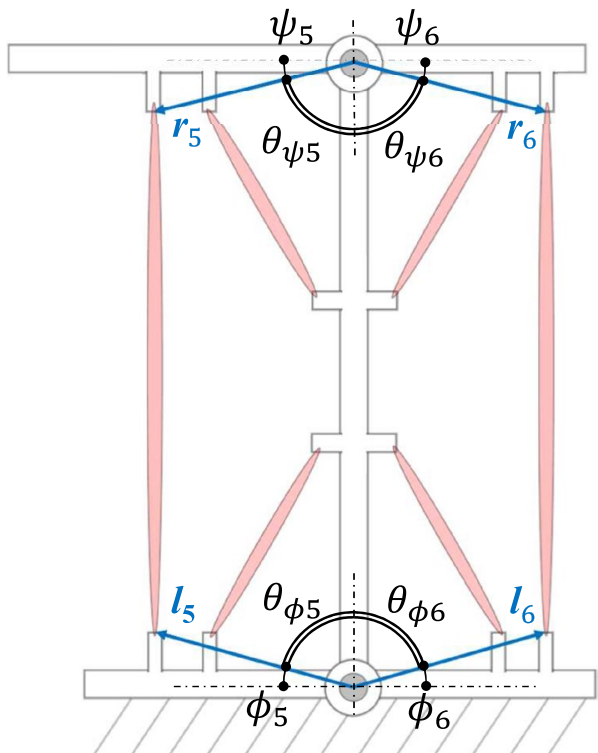

Fig. 7 Symbols used for the biarticular muscular arrangement

Table 3 Symbol list of the biarticular muscular arrangement

\begin{tabular}{|c|c|}
\hline Symbol & Meaning \\
\hline$\tilde{i}=5,6$ & The number of the biarticular muscle \\
\hline$I_{\tilde{i}}, \boldsymbol{r}_{\tilde{i}}$ & $\begin{array}{l}\text { The vectors from the rotational center of each } \\
\text { joint to the muscular fixed points }\end{array}$ \\
\hline$t_{\tilde{i}}, r_{\tilde{i}}$ & The lengths of the vector $\boldsymbol{I}_{\tilde{i}}$ and $\boldsymbol{r}_{\tilde{i}}$ \\
\hline$\phi_{\bar{i}}$ & The angle between the base and the vector $\boldsymbol{I}_{\tilde{i}}$ \\
\hline$\psi_{\tilde{i}}$ & The angle between the base and the vector $\boldsymbol{r}_{\tilde{i}}$ \\
\hline$\theta_{\tilde{\phi} \tilde{i}}$ & The angle between $\boldsymbol{r}_{\boldsymbol{i}}$ and the 1st link \\
\hline$\theta_{\psi \tilde{i}}$ & The angle between $\boldsymbol{r}_{\tilde{j}}$ and the 1st link \\
\hline
\end{tabular}

\section{Geometric condition for biarticular muscles}

This subsection contains an analysis of the geometric condition for biarticular muscles $\tilde{i}$-th $(\tilde{i}=5,6)$ in Fig. 1 . The parameters of the arrangement for the biarticular muscles are defined in polar coordinates as shown in Fig. 7. Table 3 shows the symbols of the biarticular muscular arrangement. $\boldsymbol{l}_{\tilde{i}}$ and $\boldsymbol{r}_{\tilde{i}}$ are the vectors from the rotational center of each joint to the muscular fixed points on the base/link, and the vector lengths are $l_{\tilde{i}}$ and $r_{i}$ respectively. The angle $\phi_{i}$ is defined between the base and the vector $\boldsymbol{l}_{\tilde{i}}$, and the angle $\psi_{\tilde{i}}$ is defined between the 2nd link and the vector $\boldsymbol{r}_{\tilde{i}}$.

$\theta_{\phi \tilde{i}}$ represents an angle between $\boldsymbol{l}_{\tilde{i}}$ and the 1 st link, and $\theta_{\psi \tilde{i}}$ is the angle between $\boldsymbol{r}_{\tilde{i}}$ and the 1st link. $\theta_{\phi \tilde{i}}$ and $\theta_{\psi \tilde{i}}$ change during angular motion. Moreover, the angles, $\phi_{i}$ and $\psi_{\tilde{i}}$ are constant even if the joint angles, $\theta_{1}$ and $\theta_{2}$ change. By using these defined parameters, the muscular length $q_{i}$ is expressed as follows:

$$
\begin{aligned}
q_{\tilde{i}} & =\left|\boldsymbol{l}_{\tilde{i}}-\left(\boldsymbol{r}_{\tilde{i}}+\boldsymbol{L}_{\mathbf{1}}\right)\right| \\
& =\sqrt{R-2 l_{\tilde{i}} L_{1} C_{\phi \tilde{i}}-2 r_{\tilde{i}} L_{1} C_{\psi \tilde{i}}+2 l_{\tilde{i}} r_{\tilde{i}} C_{\phi \psi \tilde{i}}},
\end{aligned}
$$

where $R=l_{\tilde{i}}^{2}+r_{\tilde{i}}^{2}+L_{1}^{2}, \quad C_{\phi \tilde{i}}=\cos \theta_{\phi \tilde{i}}, \quad C_{\psi \tilde{i}}=\cos \theta_{\psi \tilde{i}}$, $C_{\phi \psi \tilde{i}}=\cos \left(\theta_{\phi \tilde{i}}+\theta_{\psi \tilde{i}}\right)$.

Substituting Eq. (30) into Eq. (17) yields the geometric condition for the biarticular muscles. As an example, the condition for the 5 th muscle is obtained: from Eqs. (20), (21), and (22), the conditions $a_{5}>0, c_{5}>0$, $a_{5} c_{5}>b_{5}^{2}$ are obtainable for the muscle length $q_{5}$ as follows:

$$
\begin{aligned}
& a_{5}=\left.\frac{\partial^{2} q_{5}}{\partial \theta_{1}^{2}}\right|_{\theta=\theta^{*}}=\frac{-l_{5} X_{5}\left(r_{5} Y_{5}+L_{1} Z_{5}\right)}{q_{5}^{* 3}}, \\
& b_{5}=\left.\frac{\partial^{2} q_{5}}{\partial \theta_{1} \partial \theta_{2}}\right|_{\theta=\theta^{*}}=\frac{-l_{5} r_{5} X_{5} Y_{5}}{q_{5}^{* 3}}, \\
& c_{5}=\left.\frac{\partial^{2} q_{5}}{\partial \theta_{2}^{2}}\right|_{\theta=\theta^{*}}=\frac{-r_{5} Y_{5}\left(l_{5} X_{5}+L_{1} Z_{5}\right)}{q_{5}^{* 3}},
\end{aligned}
$$

where,

$$
\begin{aligned}
& X_{5}=L_{1} C_{\phi 5}^{*}-r_{5} C_{\phi \psi 5}^{*}-l_{5}, \\
& Y_{5}=L_{1} C_{\psi 5}^{*}-l_{5} C_{\phi \psi 5}^{*}-r_{5}, \\
& Z_{5}=l_{5} C_{\phi 5}^{*}+r_{5} C_{\psi 5}^{*}-L_{1} .
\end{aligned}
$$

$C_{\phi 5}^{*}, C_{\psi 5}^{*}, C_{\phi \psi 5}^{*}$ and $q_{5}^{*}$ determine the values $C_{\phi 5}, C_{\psi 5}$, $C_{\phi \psi 5}, q_{5}$ at the reference point $\boldsymbol{\theta}^{*}$ for the Taylor expansion; thus, $X, Y$, and $Z$ are constant because $\theta^{*}$ has a constant value. By substituting Eqs. (31)-(33) into Eqs. (20)-(22) (see Appendix B), the following condition that simultaneously satisfies the inequations $X_{5}>0$, $Y_{5}>0$ is obtained for the 5 th biarticular muscle:

$$
\text { Condition }(\mathrm{C}):\left\{\begin{array}{c}
L_{1} C_{\phi 5}^{*}-r_{5} C_{\phi \psi 5}^{*}-l_{5}>0, \\
\text { and } \\
L_{1} C_{\psi 5}^{*}-l_{5} C_{\phi \psi 5}^{*}-r_{5}>0 .
\end{array}\right.
$$

Next, the analysis considers the design method for the 5th biarticular muscle by expanding the preceding conditions (37) and (38). For the condition (37), the first term $L_{1} C_{\phi 5}^{*}$ implies that the length of the projection of the vector $\boldsymbol{L}_{1}$ on the vector $\boldsymbol{l}_{5}$ as shown in Fig. 8a. Next, the second term $-r_{5} C_{\phi \psi 5}^{*}$ is considered. Using the angle $\pi-\theta_{\phi 5}-\theta_{\psi 5}$ as shown in Fig. 8b, $-C_{\phi \psi 5}^{*}=-\cos \left(\theta_{\phi 5}+\theta_{\psi 5}\right)$ equals $\cos \left(\pi-\theta_{\phi 5}-\theta_{\psi 5}\right)$. 


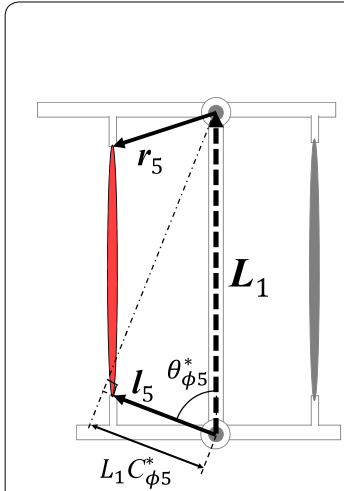

a

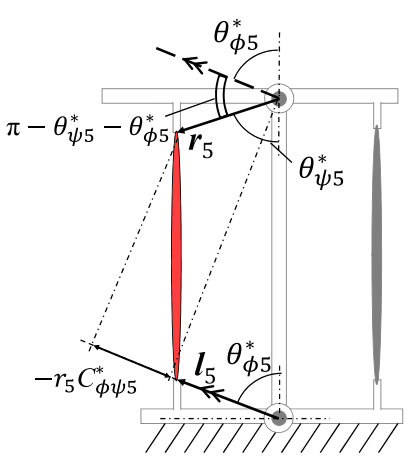

b
Fig. 8 Geometric relationship for the condition (37) of the 5th biarticular muscle

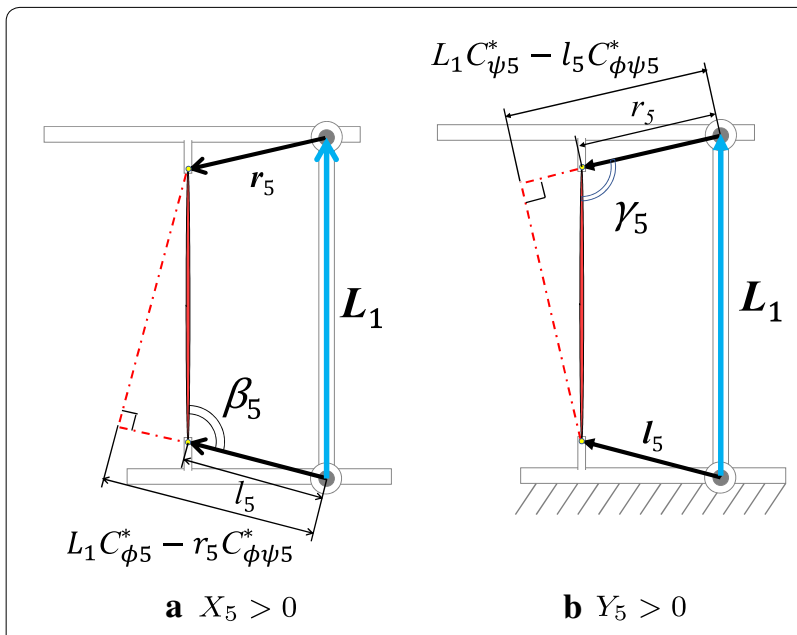

Fig. 9 Geometric conditions of the 5th biarticular muscle

Therefore, the second term $-r_{5} C_{\phi \psi 5}^{*}$ is the length of the projection of the vector $\boldsymbol{r}_{5}$ onto the vector $\boldsymbol{l}_{5}$.

As a result, the condition (37) is satisfied when the muscular length $l_{5}$ is shorter than the length $L_{1} C_{\phi 5}^{*}-r_{5} C_{\phi \psi 5}^{*}$, which the projection of the vector $L_{1}+r_{5}$ onto the vector $\boldsymbol{l}_{5}$. Namely, the geometric muscular arrangement condition is rewritten as $\beta_{5}$, which is an angle consisting of the vector $\boldsymbol{l}_{5}$ and the 5 th muscle, must be obtuse $\left(90<\beta_{5}<180\right.$ [deg] $)$ as shown in Fig. 9a.

Similarly, the condition (38) is satisfied when the length $r_{5}$ is shorter than the length $L_{1} C_{\psi 5}^{*}-l_{5} C_{\phi \psi 5}^{*}$, which is the projection of the vector $\boldsymbol{L}_{1}+\boldsymbol{l}_{5}$ onto the vector $\boldsymbol{r}_{5}$. Consequently, $\gamma_{5}$ which is an angle consisting of the vector $\boldsymbol{r}_{5}$ and the 5th muscle, must be obtuse $\left(90<\gamma_{5}<180\right.$ [deg]) when the condition (38) is satisfied, as shown in Fig. 9b.
Table 4 Parameters of muscular arrangements

\begin{tabular}{lll}
\hline & A & B \\
\hline$r_{1 \ldots 6}[\mathrm{~mm}]$ & $60,60,60,60,60,60$ & \\
$I_{1 \ldots 6}[\mathrm{~mm}]$ & $120,120,120,120,60,60$ & $0,0,0,0,0,0$ \\
$\varphi_{1 \ldots 6}[\mathrm{deg}]$ & $30,30,30,30,30,30$ & $5,5,5,5,0,0$ \\
$\psi_{1 \ldots 6}[\mathrm{deg}]$ & $5,5,5,5,30,30$ & $\mathbf{D}$ \\
\hline & $\mathbf{C}$ & \\
\hline$r_{1 \ldots 6}[\mathrm{~mm}]$ & $60,60,60,60,60,60$ & $0,0,0,0,30,30$ \\
$I_{1 \ldots 6}[\mathrm{~mm}]$ & $120,120,120,120,60,60$ & $5,5,5,5,30,30$ \\
$\varphi_{1 \ldots 6}[\mathrm{deg}]$ & $30,30,30,30,0,0$ & \\
$\psi_{1 \ldots 6}[\mathrm{deg}]$ & $5,5,5,5,0,0$ & \\
\hline
\end{tabular}

For the 6th biarticular muscle, the same result is derived. The detailed analysis of the 6th muscle is omitted due to the limitation of space.

Finally, the design for the muscular arrangement of the biarticular muscles to generate stable internal tensile forces at the desired posture is summarized as follows:

II. The geometric conditions of the biarticular muscular arrangement

The geometric muscular arrangement conditions required to satisfy $a_{5}>0, a_{6}>0, c_{5}>0, c_{5}>0$, $a_{5} c_{5}>b_{5}^{2}, a_{6} c_{6}>b_{6}^{2}$ in Eqs. (20), (21), (22) and (23) are that the angles $\beta_{\tilde{i}}$, $\gamma_{\tilde{i}}$ defined by the arrangement of the biarticular muscles $i$-th $(\tilde{i}=5,6)$ are satisfied for both of the following conditions:

$$
90<\beta_{\tilde{i}}<180 \text { and } 90<\gamma_{i}<180[\mathrm{deg}] .
$$

Table 5 Geometric conditional angles $\beta_{i}$ and $\gamma_{i}$ for each muscular arrangement

\section{Simple-joint muscles Biarticular muscles}

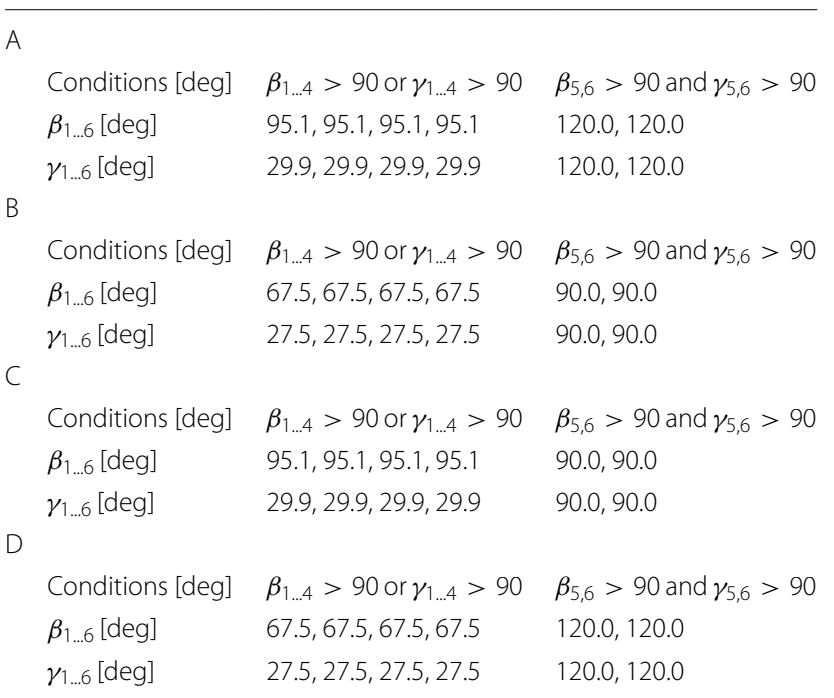




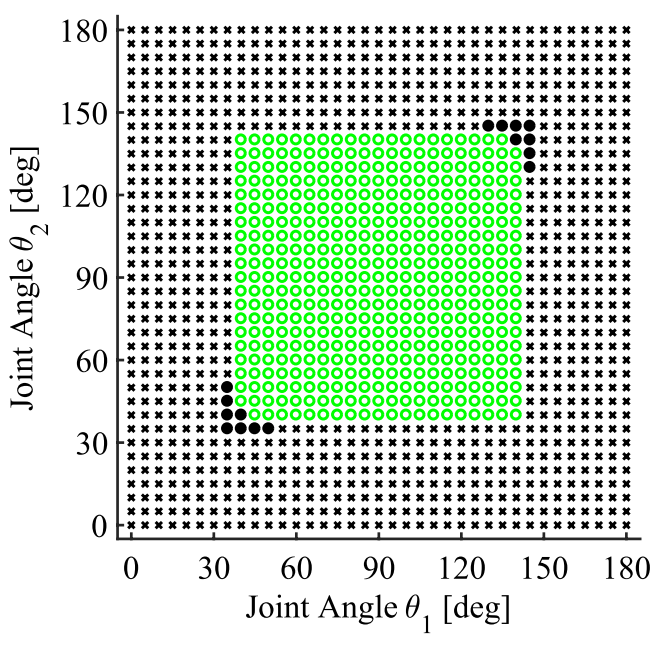

a Muscular arrangement $\mathrm{A}$

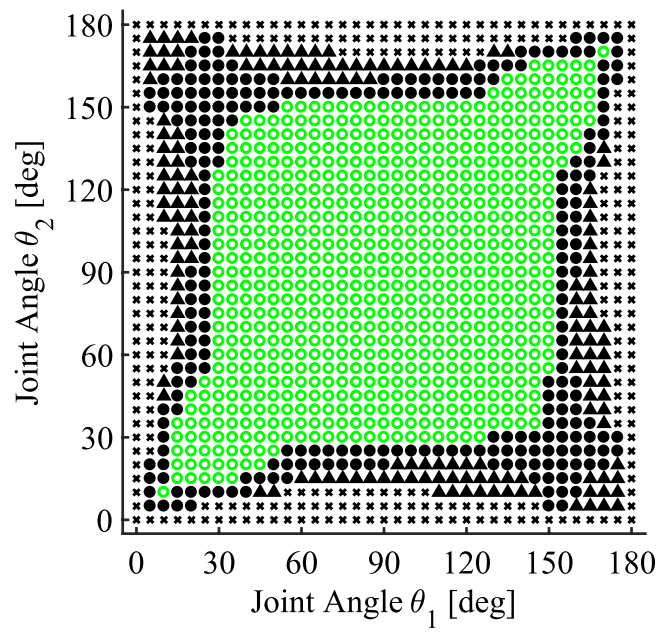

c Muscular arrangement C

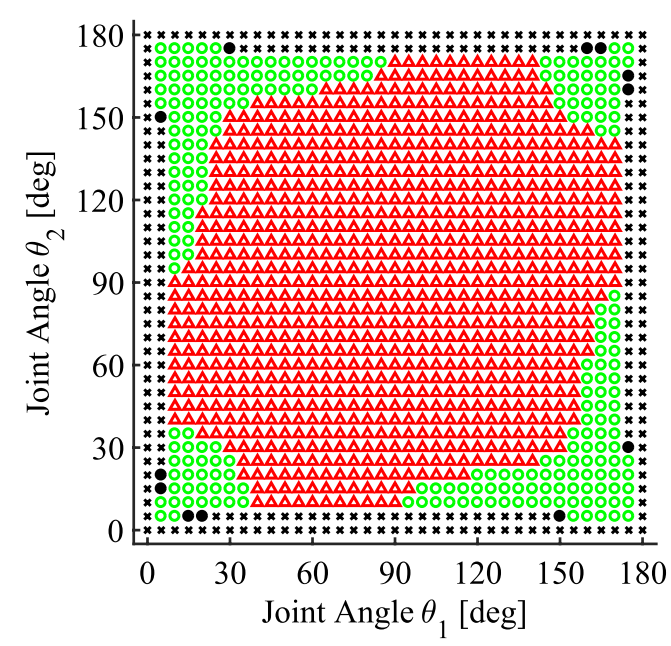

b Muscular arrangement B

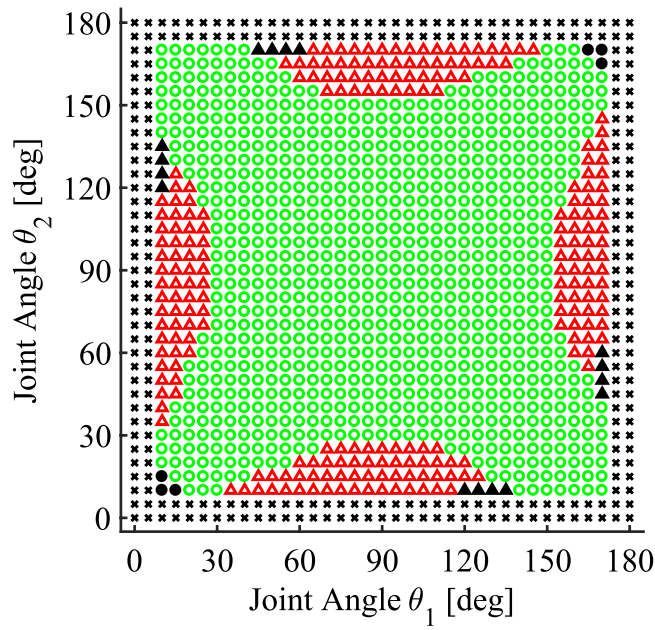

d Muscular arrangement D

Fig. 10 Minimality discrimination of the shapes of the potential at each desired posture (green circular markers indicates a local minimized potential at the posture. Red triangular markers indicates a non-minimized potential at the posture. Black circular markers indicates a local minimized potential, however, negative tensile forces are generated on the muscles. Black triangular markers indicates a non-minimized potential, and, negative tensile forces are generated on the muscles. $x$ indicates that "Vector Closure "is not satisfied)

\section{Stability verification of geometric muscular arrangement conditions}

This section verifies the conformity between the geometric conditions of the muscular arrangement and the stability of the internal tensile forces by demonstrating generated shapes of the potential for certain muscular arrangements.

The stability of the internal tensile forces is verified for the link lengths $\left(L_{1}, L_{2}\right)=(310,270)[\mathrm{mm}]$. The reference angle of the Tayler-expansion is fixed $\boldsymbol{\theta}^{*}=(90,90)^{T}[\mathrm{deg}]$. The parameters of the muscular arrangement are defined as in Figs. 5 and 7. This section demonstrates verification for the cases of the muscular arrangements A, B, C and D as represented in Table 4. Interference between the muscles and the links is ignored for the analysis.

Firstly, the conditional angles $\beta_{i}$ and $\gamma_{i}$ for the reference angle $\boldsymbol{\theta}^{*}=(90,90)^{T}[\mathrm{deg}]$ in the muscular arrangements A - D are represented in Table 5 . The muscular arrangement A satisfies the stable conditions $\beta_{1 \ldots 4}>90$ [deg] or $\gamma_{1 \ldots 4}>90\left[\mathrm{deg}\right.$ ], $\beta_{5,6}>90$ [deg] and $\gamma_{5,6}>90$ [deg] for all muscles. However, the muscular arrangement B does not satisfy all the conditions. The muscular arrangement $\mathrm{C}$ only satisfies the conditions for the simple-joint muscles $1-4$, however, it does not satisfy the conditions for the 


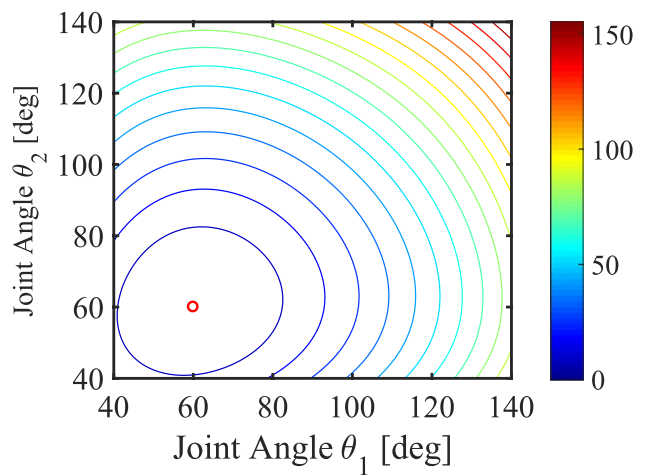

a $\boldsymbol{\theta}_{d}=(60,60)^{T}[\mathrm{deg}]$

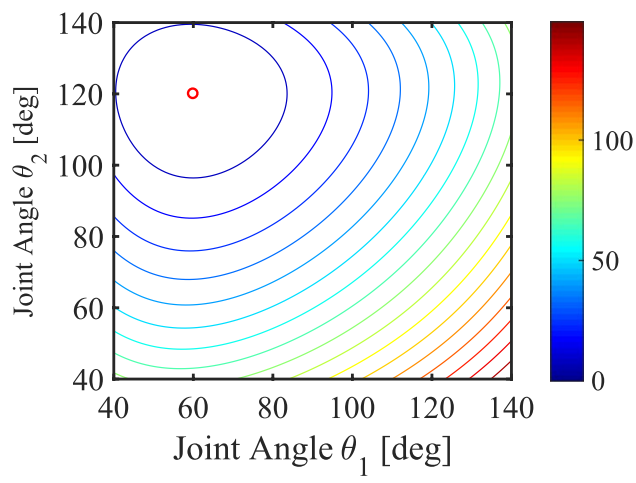

c $\boldsymbol{\theta}_{d}=(60,120)^{T}[\mathrm{deg}]$

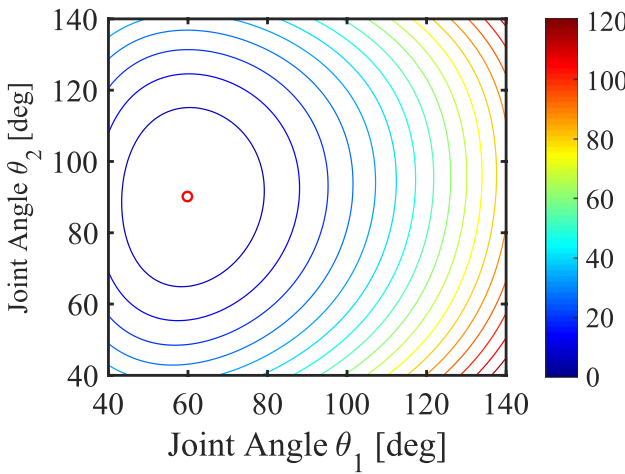

b $\boldsymbol{\theta}_{d}=(60,90)^{T}[\mathrm{deg}]$

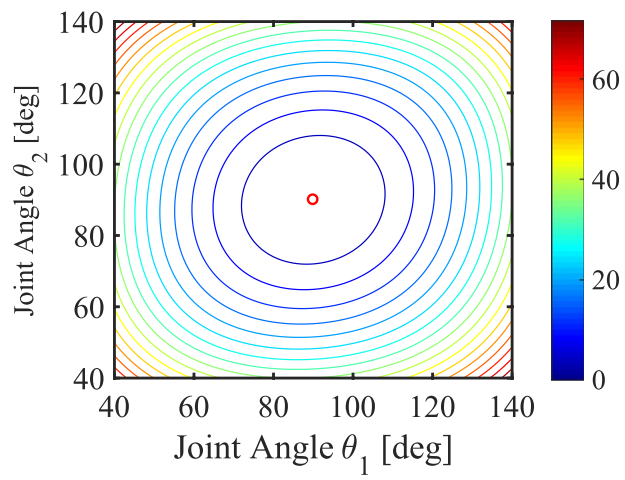

d $\boldsymbol{\theta}_{d}=(90,90)^{T}[\mathrm{deg}]$

Fig. 11 Potentials of the muscular arrangement A

biarticular muscles 5 and 6 . Contrary to arrangement $C$, the muscular arrangement $D$ satisfies the conditions for the biarticular muscles 5 and 6, however, it does not satisfy the conditions for the simple-joint muscles 1-4.

Next, for each of the cases of the muscular arrangements A-D, the stability of internal tensile forces at the desired posture is numerically examined to verify the effectiveness of the analyzed conditions. Using Eqs. (11) and (12), Fig. 10 describes the minimization of the potential at the desired posture for every five degrees ranging $0<\theta_{1}<180$ [deg], $0<\theta_{2}<180$ [deg]. The arbitrary vector $\boldsymbol{k}_{\boldsymbol{e}}$ on Eq. (6) is determined as $\boldsymbol{k}_{\boldsymbol{e}}=(1, \ldots, 1)^{T}$. In Fig. 10, the green circular markers indicate that the shape of the potential for the desired posture is a local minimum at the posture. The red triangular markers indicate that the shape of the potential for the desired posture is not a local minimum at the posture. The black circular markers indicate that the potential is a local minimum, however, it does not satisfy the assumption outlined in the second section because negative tensile forces (pushing forces) are generated. The black triangular markers indicate that the potential is not a local minimum, and it does not satisfy the assumption because negative tensile forces are generated. For the cases in which negative tensile forces are generated at black circular markers and black triangular markers, the input force may be positive by selecting a suitable arbitrary vector $\boldsymbol{k}_{\boldsymbol{e}}$; however, this section does not examine vector selection. The cross makers $\times$ indicate that the muscular arrangement at the posture does not satisfy "Vector Closure [15] ", namely, does not have an antagonist muscular arrangement at the posture.

Figure 10a shows that the muscular arrangement A generates stable potentials in the range of approximately $30<\theta_{1}<150,30<\theta_{2}<150$ [deg]. The muscular arrangement $B$ generates unstable potentials in the vicinity of the posture $\boldsymbol{\theta}_{\boldsymbol{d}}=(90,90)[\mathrm{deg}]$ as shown in Fig. 10b, because it does not satisfy all the conditions. 


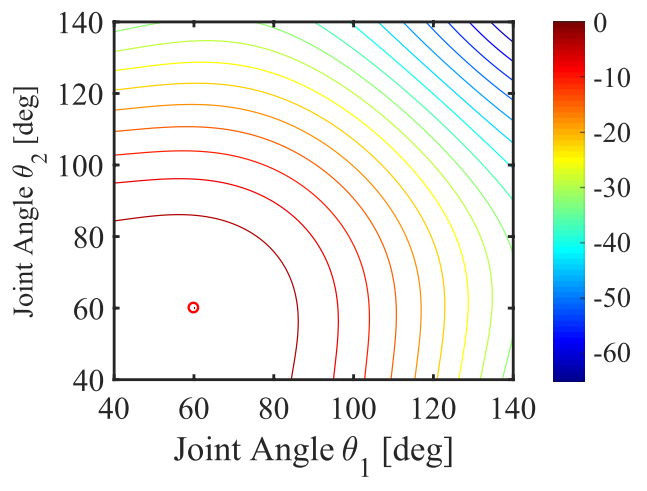

a $\boldsymbol{\theta}_{d}=(60,60)^{T}[\mathrm{deg}]$

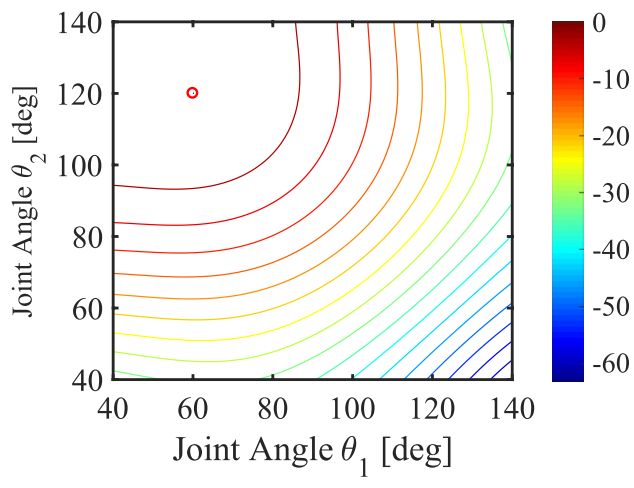

C $\boldsymbol{\theta}_{d}=(60,120)^{T}[\mathrm{deg}]$

Fig. 12 Potentials of the muscular arrangement $B$

The muscular arrangements $C$ and $D$ generate stable potentials in the vicinity of the posture $\boldsymbol{\theta}_{\boldsymbol{d}}=(90,90)$ [deg] as shown in Fig. 10c and d. The ranges that satisfy Vector Closure for the muscular arrangements $\mathrm{C}$ and $\mathrm{D}$ are more extensive than that those of the muscular arrangement A because of the absence of an offset with either the simple-joint or the biarticular muscles which expands the range. The muscular arrangement A generates stable potentials when it satisfies Vector Closure. For the other cases of the muscular arrangements i.e. $C$ and $D$, unstable potentials are generated at some postures although Vector Closure is satisfied. The ranges that generate stable potentials for the muscular arrangements $C$ and $D$ are more extensive than that of the muscular arrangement $\mathrm{A}$, even though these muscular arrangements do not satisfy some geometric conditions. This is because the muscular arrangements satisfy the condition (12) for generating a local minimum potential at those postures by strongly satisfying the conditions (29) or (39) in some muscles.

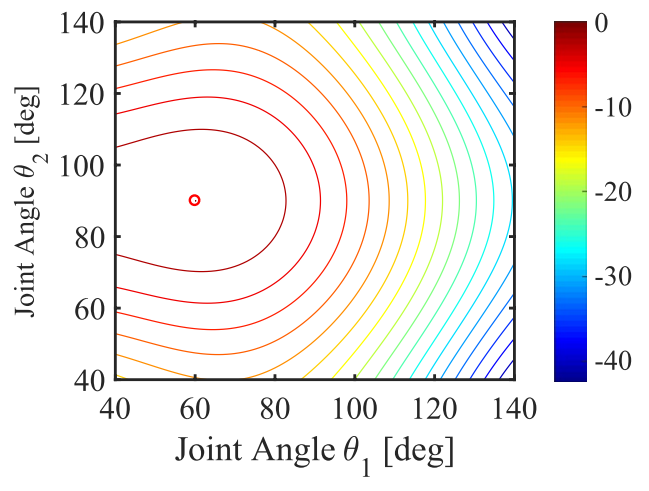

b $\boldsymbol{\theta}_{d}=(60,90)^{T}[\mathrm{deg}]$

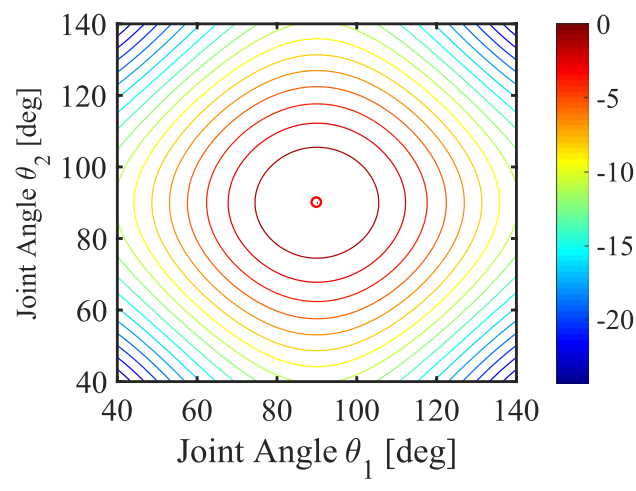

d $\boldsymbol{\theta}_{d}=(90,90)^{T}[\mathrm{deg}]$
Additionally, the shapes of the potential for the muscular arrangements A-D at $\boldsymbol{\theta}_{\boldsymbol{d}}=(60,60),(60,90)$, $(60,120),(90,90)[\mathrm{deg}]$ are demonstrated as Figs. 11, 12, 13,14 . In these figures, the red circular marker identifies the desired posture $\boldsymbol{\theta}_{\boldsymbol{d}}$. The shapes of the potential at $\boldsymbol{\theta}_{\boldsymbol{d}}=(60,60),(60,90),(60,120)[\mathrm{deg}]$ and the shapes at $\boldsymbol{\theta}_{\boldsymbol{d}}=(120,120),(90,60),(120,60)$ [deg] are symmetric, because the muscular arrangements are vertically and horizontally symmetric and the potential is static.

The potentials of the muscular arrangement A have stable shapes for all cases as shown in Fig. 11. The potentials of the muscular arrangement $B$ have unstable shapes for all cases as shown in Fig. 12. The potentials of muscular arrangements $C$ and D have stable shapes for all cases as shown in Figs. 13 and 14, however, the gradients are gentler than that of the muscular arrangement $\mathrm{A}$. This is because that the muscular arrangement $C$ does not satisfy the conditions of the biarticular muscles, the muscular arrangement D does not satisfy the conditions of the 


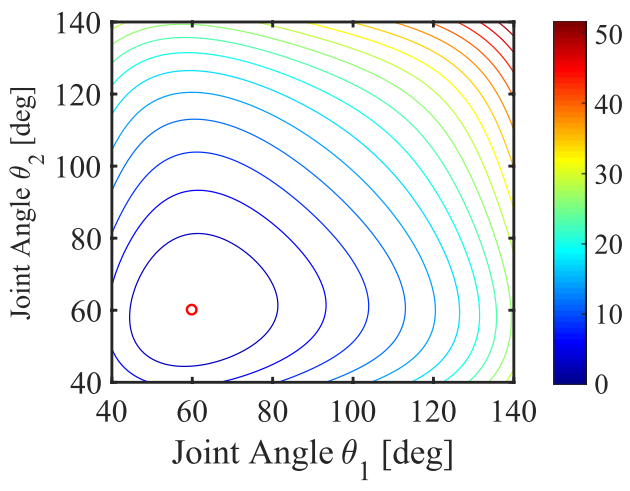

a $\boldsymbol{\theta}_{d}=(60,60)^{T}[\mathrm{deg}]$

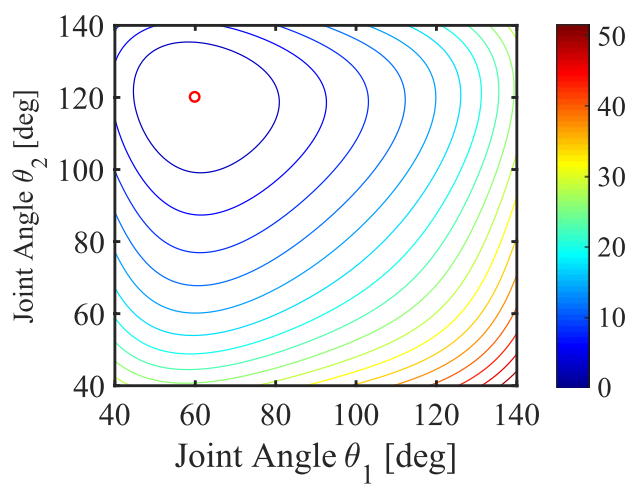

c $\boldsymbol{\theta}_{d}=(60,120)^{T}[\mathrm{deg}]$

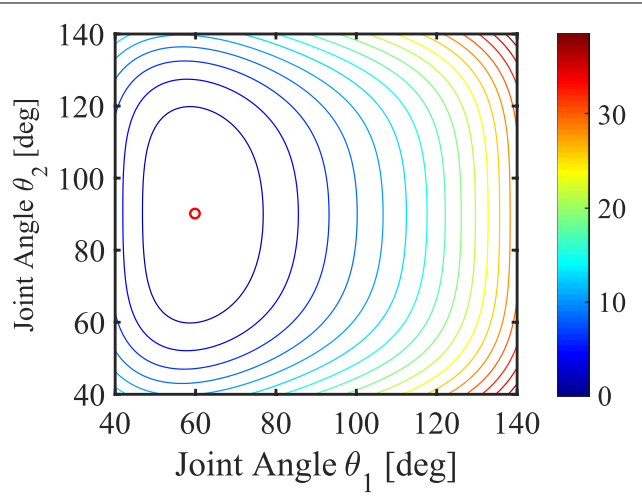

b $\boldsymbol{\theta}_{d}=(60,90)^{T}[\mathrm{deg}]$

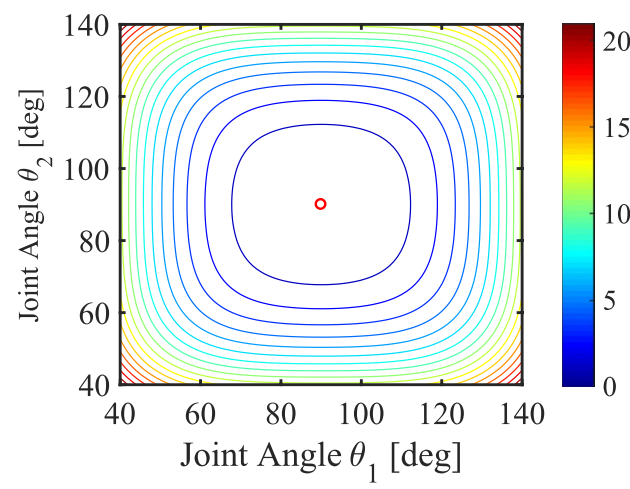

d $\boldsymbol{\theta}_{d}=(90,90)^{T}[\mathrm{deg}]$

Fig. 13 Potentials of the muscular arrangement $C$

simple-joint muscles; therefore those muscles decrease the stability.

Even though the reference angle of the Tayler-expansion is $\boldsymbol{\theta}^{*}=(90,90)[\mathrm{deg}]$, the stable potential generates an extensive postural range that is not confined to the vicinity of $\boldsymbol{\theta}^{*}$. Some cases generate a stable potential at the desired posture even when the geometric conditions are not satisfied. This is because the conditions are sufficient for generating a stable potential.

Consequently, the aforementioned results indicate that the stable internal tensile forces are generated when the musculoskeletal structure satisfies the geometric conditions presented in this study.

\section{Conclusion}

This study has analyzed the geometric muscular arrangement condition based on the generation of stable internal tensile forces for a musculoskeletal manipulator with two-link and six-muscle. The geometric muscular arrangement conditions have been derived from the mathematical conditions in which the potential is minimum at the desired posture. According to the results, the stability of the internal tensile forces is conditioned on the angles between the line from the driven joint to the muscular fixed point and the line on the muscle.

The condition is very simple and sufficient to form a stable potential that is generated by the internal tensile forces of the muscles. By designing the muscular 


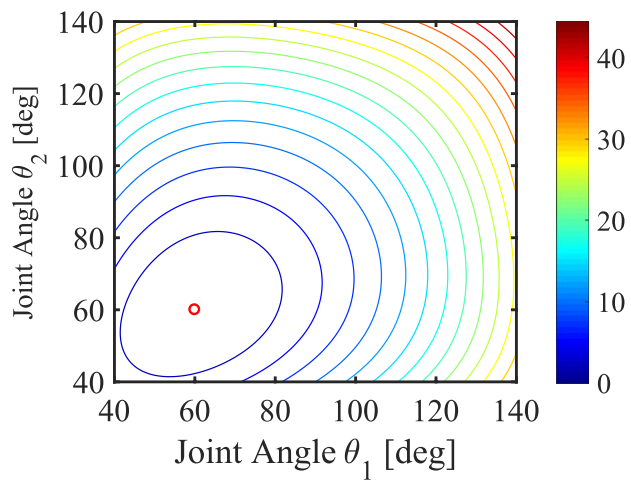

a $\boldsymbol{\theta}_{d}=(60,60)^{T}[\mathrm{deg}]$

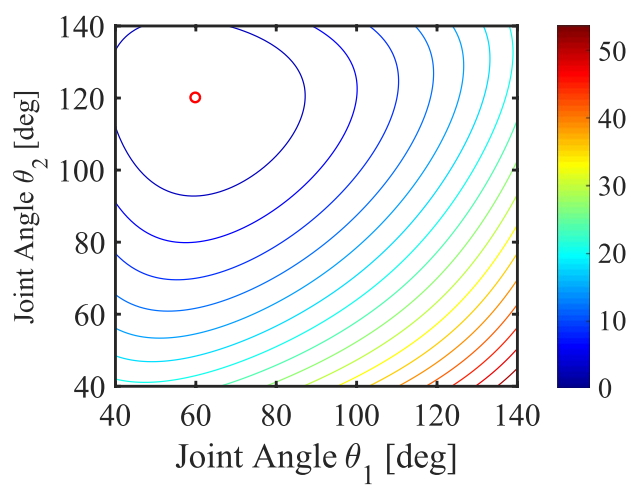

c $\boldsymbol{\theta}_{d}=(60,120)^{T}[\mathrm{deg}]$

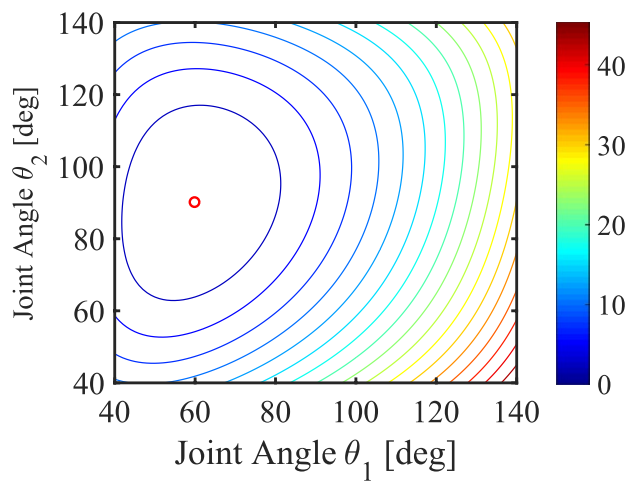

b $\boldsymbol{\theta}_{d}=(60,90)^{T}[\mathrm{deg}]$

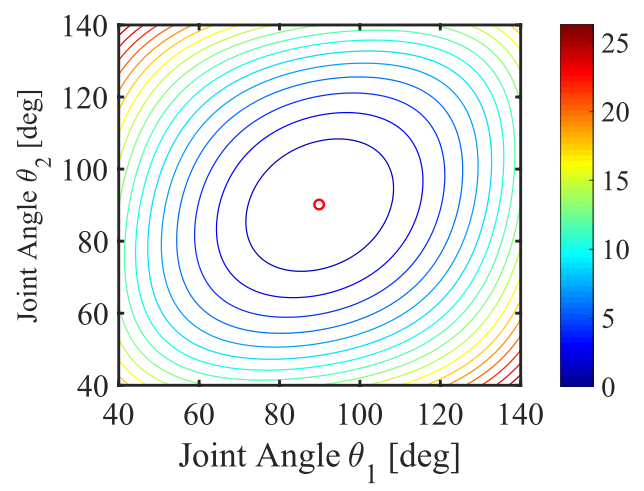

d $\boldsymbol{\theta}_{d}=(90,90)^{T}[\mathrm{deg}]$

Fig. 14 Potentials of the muscular arrangement $D$

arrangement to satisfy the conditions, the stability of the internal tensile forces is ensured, and consequently, a control method utilizing the characteristics of the internal tensile force is stably implemented. The acquired result is valuable because the internal tensile force of the unstable muscular arrangement certainly influences the system's stability, even for the musculoskeletal system controlled by using a typical feedback method.

The future direction for this work is to expand the analysis to structures with more joints and muscles. Additionally, future works will involve the analysis of the condition whereby the vector $\boldsymbol{k}_{\boldsymbol{e}}$ that affects the stability of the internal tensile forces is considered.

\section{Acknowledgements}

Not applicable.

\section{Authors' contributions}

$\mathrm{HO}, \mathrm{HK}, \mathrm{KT}$, and YM contributed to the concepts and the analysis design. $\mathrm{HO}$ carried out the analysis and drafted the manuscript. HK advised the analysis and revised the manuscript. All authors read and approved the final manuscript.

\section{Funding}

This work was supported by the Japanese Society for the Promotion of Science, for Scientific Research (C), (Grant Number 26330303), 2014-2016.

\section{Availability of data and materials}

Not applicable.

\section{Competing interests}

The authors declare that they have no competing interests.

\section{Author details}

1 Department of Mechanical Engineering, Faculty of Engineering, SanyoOnoda City University, 1-1-1, Daigaku-Dori, Sanyo-Onoda, Yamaguchi 756-0884, Japan. ${ }^{2}$ Department of Intelligent Mechanical Engineering, Faculty of Engineering, Fukuoka Institute of Technology, 3-30-1, Wajiro-higashi, Fukuoka 811-0295, Higashi-ku, Japan. ${ }^{3}$ Department of Mechanical Engineering, Faculty of Engineering, Kyushu University, 744, Moto'oka, Nishi-ku, Fukuoka 819-0395, Japan. ${ }^{4}$ Department of Robotics, Faculty of Engineering, Kindai University, 1, Takaya Umenobe, Higashi-Hiroshima, Hiroshima 739-2116, Japan. 


\section{Appendix A: Proof of stable condition in "Stability of the internal tensile forces" section}

Section 3 describes the sufficient stable condition i.e., all the values in the vector $\boldsymbol{u}$ and the matrix $\boldsymbol{N}$ shown on Eqs. (18) and (19) are positive. Additionally, it describes that the positivity of the value in the 5th row and 6th column in the matrix $\boldsymbol{N}$ does not need to be considered when the other value is positive. This appendix demonstrates that $a_{5} c_{6}+a_{5} c_{5}-2 b_{5} b_{6}>0$ does not have to be considered when Eqs. (20)-(23) are satisfied.

Where Eqs. (22) and (23) multiply both sides of each equation and the following equation is obtained:

$$
a_{5} a_{6} c_{5} c_{6}>b_{5}^{2} b_{6}^{2} .
$$

When Eqs. (20) and (21) are satisfied, $a_{5}>0, a_{6}>0$, $c_{5}>0, c_{6}>0$, thus Eq. (40) is obtained:

$$
\sqrt{a_{5} a_{6} c_{5} c_{6}}>\left|b_{5} b_{6}\right| \text {. }
$$

Let $\left(\sqrt{a_{5} c_{5}}+\sqrt{a_{6} c_{6}}\right)^{2}>0$ satisfy Eqs. (20) and (21). Therefore, the equation is rewritten as follows:

$$
a_{5} c_{5}+a_{6} c_{6}>2 \sqrt{a_{5} a_{6} c_{5} c_{6}} .
$$

From Eqs. (41) and (42),

$$
\begin{aligned}
& a_{5} c_{5}+a_{6} c_{6}>2 \sqrt{a_{5} a_{6} c_{5} c_{6}}>2\left|b_{5} b_{6}\right|, \\
& a_{5} c_{5}+a_{6} c_{6}-2\left|b_{5} b_{6}\right|>0 .
\end{aligned}
$$

The equation describes satisfying $a_{5} c_{6}+a_{5} c_{5}-2 b_{5} b_{6}>0$ when Eqs. (20)-(23) are satisfied.

Therefore, the positivity of the value in the 5 th row and 6th column in the matrix $N$ does not need to be considered when Eqs. (20)-(23) are satisfied. As a result, only Eqs. (20)-(23) represent the sufficient stable condition.

\section{Appendix B: Analysis of Geometric conditions for the biarticular muscular arrangement}

This appendix focuses on the analysis of the geometric condition for the 5th biarticular muscular arrangement. The stable conditions for this biarticular muscle are $a_{5}>0, c_{5}>0, a_{5} c_{5}>b_{5}^{2}$ from Eqs. (20)-(22). Eq. (22) is rewritten as the following when Eqs. (31)-(33) is substituted:

$$
\frac{l_{5} r_{5} L_{1} X_{5} Y_{5} Z_{5}\left(l_{5} X_{5}+r_{5} Y_{5}+L_{1} Z_{5}\right)}{q_{5}^{* 6}}>0 .
$$

In addition, Eq. (30) is rewritten as the following when Eqs. (34)-(36) is substituted:

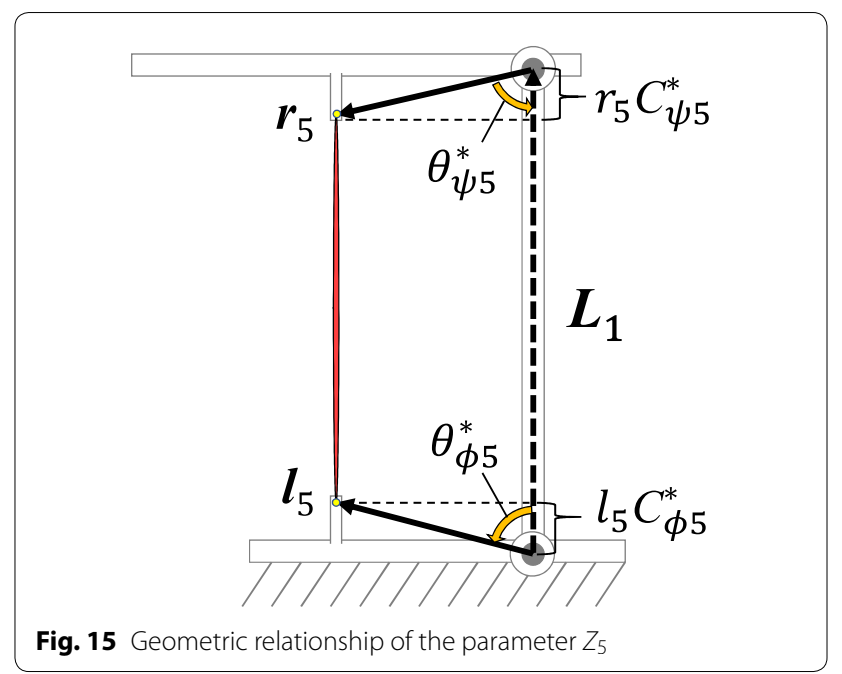

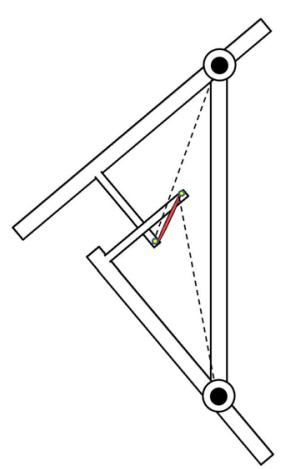

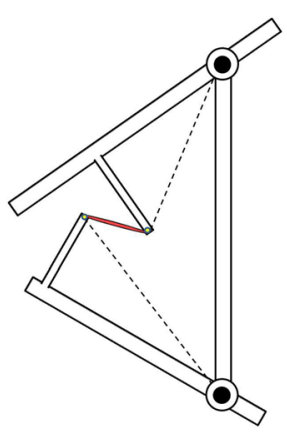

b
Fig. 16 The structures for the case of $Z_{5}>0$

$$
l_{5} X_{5}+r_{5} Y_{5}+L_{1} Z_{5}=-q_{5}^{* 2} .
$$

Therefore, Eq. (45) is rewritten as the following by replacing Eq. (46):

$$
-\frac{l_{5} r_{5} L_{1} X_{5} Y_{5} Z_{5}}{q_{5}^{* 4}}>0 .
$$

Let $q_{5}^{*}, l_{5}, r_{5}, L_{1}>0$, hence the next equation should be satisfied to fulfill Eq. (47):

$$
X_{5} Y_{5} Z_{5}<0 .
$$

Next, the geometric relationship for the parameter $Z_{5}$ defined in Eq. (36) is considered. $l_{5} C_{\phi 5}^{*}, r_{5} C_{45}^{*}$ in $Z_{5}$ are the projected lengths of the vector $\boldsymbol{l}_{5}$ and $\boldsymbol{r}_{5}$ on the link vector $\boldsymbol{L}_{1}$ similar to Fig. 15 . When $Z_{5}>0$, for the based position $\boldsymbol{\theta}^{*}$ in the Taylor-expansion, the structure interacts with each link/base similar to Fig. 16a or differ substantially from the human structure similar to Fig. 16b. 
Therefore in the analysis, only $Z_{5}<0$ is assumed. When $Z_{5}<0, X_{5}$ and $Y_{5}$ have the same sign from Eq. (48).

First, the case of $X_{5}<0$ and $Y_{5}<0$ is considered. Eq. (31) is substituted for Eq. (20) and the following condition is obtained from $-l_{5} X_{5}>0$ :

$$
r_{5} Y_{5}>-L_{1} Z_{5} \text {. }
$$

Similarly, Eq. (33) is substituted for Eq. (21) and the following condition is obtained from $-r_{5} Y_{5}>0$ :

$$
l_{5} X_{5}>-L_{1} Z_{5} \text {. }
$$

Let $r_{5} Y_{5}<0, l_{5} X_{5}<0,-L_{1} Z_{5}>0$ from $X_{5}<0, Y_{5}<0$, $Z_{5}<0$. Thus, the conditions (49) and (50) are contradicted with the supposition $X_{5}<0$ and $Y_{5}<0$. Therefore, the case of $X_{5}<0$ and $Y_{5}<0$ is not satisfied.

Next, the case of $X_{5}>0$ and $Y_{5}>0$ is considered. Eqs. (31) and (33) are substituted for Eqs. (20) and (21) and the following conditions are obtained:

$$
\begin{aligned}
& r_{5} Y_{5}<-L_{1} Z_{5}, \\
& l_{5} X_{5}<-L_{1} Z_{5} .
\end{aligned}
$$

Considering Eq. (46), we obtain the following conditions by adding $-l_{5} X_{5}-r_{5} Y_{5}$ to both sides in Eqs. (51) and (52):

$$
\begin{aligned}
& -l_{5} X_{5}<-\left(l_{5} X_{5}+r_{5} Y_{5}+L_{1} Z_{5}\right) \\
& -r_{5} Y_{5}<-\left(l_{5} X_{5}+r_{5} Y_{5}+L_{1} Z_{5}\right) .
\end{aligned}
$$

In these equations, $-\left(l_{5} X_{5}+r_{5} Y_{5}+L_{1} Z_{5}\right)$ is $q_{5}^{* 2}$ from Eq. (46). Therefore, the conditions are rewritten as follows:

$$
\begin{aligned}
& -l_{5} X_{5}<q_{5}^{* 2} \\
& -r_{5} Y_{5}<q_{5}^{* 2}
\end{aligned}
$$

Let $-l_{5} X_{5}<0$ and $-r_{5} Y_{5}<0$ from $X_{5}>0, Y_{5}>0$ and $q_{5}^{* 2}>0$. Consequently, these conditions are always satisfied when $X_{5}>0, Y_{5}>0$.

As a result, the stable conditions (20)-(23) are rewritten as Eqs. (37) and (38). The geometric condition for the 6th biarticular muscular arrangement can be analyzed in a similar manner.
Received: 29 September 2019 Accepted: 12 March 2020

Published online: 30 March 2020

\section{References}

1. Bernstein NA (1935) The problem of interrelation between coordination and localization. Arch Biol Sci 38:1-35

2. Bizzi E, Accornero N, Chapple W, Horgan N (1984) Posture control and trajectory formation during arm movement. J Neurosci 4:2738-2744

3. Kawato M, Furukawa K, Suzuki R (1987) A hierarchical neural-network model for control and learning of voluntary movement. Biol Cybern 57:169-185

4. Kumamoto M, Oshima T, Yamamoto T (1994) Control properties induced by the existence of antagonistic pairs of bi-articular muscles-mechanical engineering model analyses. Hum Mov Sci 13:611-634

5. Kozuki T, Toshinori H, Shirai T, Nakashima S, Asano Y, Kakiuchi Y, Okada K, Inaba M. Skeletal structure with artificial perspiration for cooling by latent heat for musculoskeletal humanoid kengoro. In: Proceedings of IEEE/ RSJ international conference on intelligent robots and systems. 2016. p. $2135-2140$

6. Koganezawa K, Shimizu Y. Stiffness control of tendon driven multi-dof joint by actuator with non linear elastic system (ANLES). In: Proceedings of international conference on advanced robotics, 2003. p. 1184-9

7. Mitsuda T, Kawamura S, Uemura M, Sekimoto M (2011) Diagonalization of arm kinematics by the use of bi-articular muscles. SICE J Control Measur Syst Integrat 4(2):114-119

8. Ozawa R, Kobayashi H, Hashirii K (2014) Analysis, classification and design of tendon-driven mechanisms. IEEE Trans Robot 30(2):396-410

9. Hitzmann A, Masuda H, Ikemoto S, Hosoda K (2018) Anthropomorphic musculoskeletal 10 degrees-of-freedom robot arm driven by pneumatic artificial muscles. Adv Robot 32(15):1-14

10. Shin H, Ikemoto S, Hosoda K (2018) Constructive understanding and reproduction of functions of gluteus medius by using a musculoskeletal walking robot. Adv Robot 32(4):202-214

11. Kino H, Yahiro T, Taniguchi S, Tahara K (2009) Sensorless position control using feedforward internal force for completely restrained parallel-wire driven systems. IEEE Trans Robot 25(2):467-474

12. Kino H, Kikuchi S, Matsutani Y, Tahara K, Nishiyama T (2013) Numerical analysis of feedforward position control for non-pulley-musculoskeletal system: a case study of macular arrangements of a two-link planar system with six muscles. Adv Robot 27(16):1235-1248

13. Matsutani Y, Tahara K, Kino H, Ochi H, Yamamoto M. Set-point control of a musculoskeletal arm by the complementary combination of a feedforward and feedback manner. In: Proceedings of IEEE international conference on robotics and Automation. 2014. p. 5908-14.

14. Kino H, Ochi H, Matsutani Y, Tahara K (2017) Sensorless point-to-point control for a musculoskeletal tendon-driven manipulator: analysis of a two-dof planar system with six tendons. Adv Robot 31(16):851-864

15. Kino H, Yahiro T, Takemura F, Morizono T (2007) Robust pd control using adaptive compensation for completely restrained parallel-wire driven robots: translational systems using the minimum number of wires under zero-gravity condition. IEEE Trans Robot 23(4):803-812

\section{Publisher's Note}

Springer Nature remains neutral with regard to jurisdictional claims in published maps and institutional affiliations. 